\title{
Novel Functionalized Trisubstituted Allylboronates via Hosomi-Miyaura Borylation of Functionalized Allyl Acetates.
}

P. Veeraraghavan Ramachandran*, Debarshi Pratihar, Debanjan Biswas, Amit Srivastava and M. Venkat Ram Reddy

Herbert. C. Brown Center for Borane Research, 560 Oval Drive, Department of Chemistry, Purdue University, West Lafayette, IN 47907-2084.

Table of contents

\begin{tabular}{|c|c|}
\hline Contents & Page Numbers \\
\hline NMR Data & S2-S5 \\
\hline Spectra & S6-S40 \\
\hline
\end{tabular}




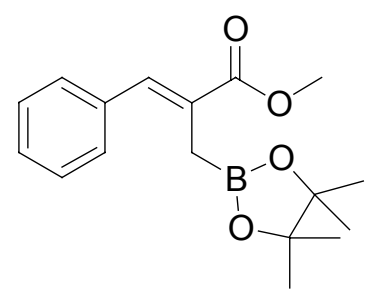

8a.

${ }^{1} \mathrm{H}$ NMR $\left(300 \mathrm{MHz}, \mathrm{CDCl}_{3}\right) \delta(\mathrm{ppm}): 7.67(\mathrm{~s}, 1 \mathrm{H}), 7.41-7.26(\mathrm{~m}, 5 \mathrm{H}), 3.80(\mathrm{~s}, 3 \mathrm{H})$, $2.14(\mathrm{~s}, 2 \mathrm{H}), 1.24(\mathrm{~s}, 12 \mathrm{H}) ;{ }^{13} \mathrm{C}$ NMR $\left(75 \mathrm{MHz}, \mathrm{CDCl}_{3}\right) \delta(\mathrm{ppm}): 169.1,137.6,136.2$, 130.2, 129.4, 128.3, 128.1, 83.5, 52.1, 24.7; ${ }^{11} \mathrm{~B}$ NMR $\delta(\mathrm{ppm}): 33$; EI-MS: $\mathrm{m} / \mathrm{z} 271$ $(\mathrm{M})^{+}, 59\left[\left(\mathrm{CO}_{2} \mathrm{CH}_{3}\right)^{+}, 100 \%\right]$; CI-MS: $\mathrm{m} / \mathrm{z} 303(\mathrm{M}+\mathrm{H})^{+}, 271\left[\left(\mathrm{M}+\mathrm{H}-\mathrm{HOCH}_{3}\right)^{+}, 100 \%\right]$; HRMS-CI: 303.1768 (calcd.), 303.1763 (actual).

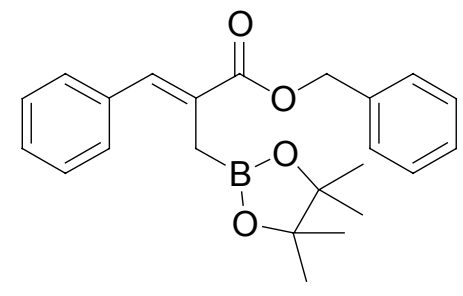

$8 b$.

${ }^{1} \mathrm{H}$ NMR $\left(300 \mathrm{MHz}, \mathrm{CDCl}_{3}\right) \delta(\mathrm{ppm}): 7.73(\mathrm{~s}, 1 \mathrm{H}), 7.44-7.29(\mathrm{~m}, 10 \mathrm{H}), 5.26(\mathrm{~s}, 2 \mathrm{H}), 2.18$ (s, 2H), 1.19 (s, 12H); ${ }^{13} \mathrm{C}$ NMR $\left(75 \mathrm{MHz}, \mathrm{CDCl}_{3}\right) \delta(\mathrm{ppm}): 168.5,137.9,136.2,130.2$, 129.4, 128.5, 128.3, 128.1, 83.5, 66.7, 24.7; ${ }^{11}$ B NMR $\delta$ (ppm): 33; EI-MS: m/z 278 (MC7H6) ${ }^{+}, 91\left[\left(\mathrm{C}_{7} \mathrm{H}_{7}\right)^{+}, 100 \%\right]$; CI-MS: m/z 379; HRMS-CI: 379.2081 (calcd.), 379.2071 (actual).

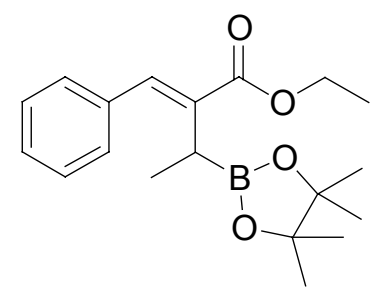

8c.

${ }^{1} \mathrm{H}$ NMR $\left(300 \mathrm{MHz}, \mathrm{CDCl}_{3}\right) \delta(\mathrm{ppm}): 7.64(\mathrm{~s}, 1 \mathrm{H}), 7.42-7.30(\mathrm{~m}, 5 \mathrm{H}), 4.26(\mathrm{~m}, 2 \mathrm{H}), 2.49$ $(\mathrm{q}, J=7.30 \mathrm{~Hz}, 1 \mathrm{H}), 1.35(\mathrm{t}, J=7.12 \mathrm{~Hz}, 3 \mathrm{H}), 1.26(\mathrm{~s}, 6 \mathrm{H}), 1.22(\mathrm{~s}, 6 \mathrm{H}) ;{ }^{13} \mathrm{C}$ NMR $(75$ $\left.\mathrm{MHz}, \mathrm{CDCl}_{3}\right) \delta(\mathrm{ppm}): 168.3,137.3,136.3,129.2,128.4,128.0,83.3 ., 60.9,24.9,24.7$, 
15.6, 14.4; ${ }^{11} \mathrm{~B}$ NMR $\delta$ (ppm): 33; EI-MS: m/z $330(\mathrm{M})^{+}, 315$ [(M-CH$\left.)^{+}, 100 \%\right]$; CI-MS: m/z $331(\mathrm{M}+\mathrm{H})^{+}$; HRMS-EI: 330.2002 (calcd.), 330.1997 (actual).

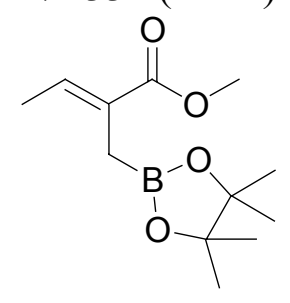

9a.

${ }^{1} \mathrm{H}$ NMR $\left(300 \mathrm{MHz}, \mathrm{CDCl}_{3}\right) \delta(\mathrm{ppm}): 6.82(\mathrm{q}, J=7.02 \mathrm{~Hz}, 1 \mathrm{H}), 3.70(\mathrm{~s}, 3 \mathrm{H}), 1.85$ (s, $2 \mathrm{H}), 1.77(\mathrm{~d}, J=7.05 \mathrm{~Hz}, 2 \mathrm{H}), 1.23(\mathrm{~s}, 12 \mathrm{H}) ;{ }^{13} \mathrm{C} \mathrm{NMR}\left(75 \mathrm{MHz}, \mathrm{CDCl}_{3}\right) \delta(\mathrm{ppm})$ : 168.5, 137.8, 135.7, 130.0, 83.3, 51.6, 24.6, 14.5; ${ }^{11} \mathrm{~B}$ NMR $\delta$ (ppm): 32; EI-MS: m/z 240 $(\mathrm{M})^{+}$, 54; CI-MS: m/z $241(\mathrm{M}+\mathrm{H})^{+}$; HRMS-EI: 240.1533 (calcd.), 240.1541 (actual).

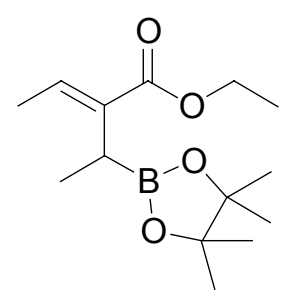

$9 c$.

${ }^{1} \mathrm{H}$ NMR (300 MHz, $\left.\mathrm{CDCl}_{3}\right) \delta(\mathrm{ppm}): 6.81(\mathrm{q}, J=7.11 \mathrm{~Hz}, 1 \mathrm{H}), 4.17(\mathrm{~m}, 2 \mathrm{H}), 2.15$ (q, $J$ $=7.41 \mathrm{~Hz}, 1 \mathrm{H}), 1.81(\mathrm{~d}, J=7.14 \mathrm{~Hz}, 3 \mathrm{H}), 1.27(\mathrm{t}, J=3.52 \mathrm{~Hz}, 9 \mathrm{H}), 1.23(\mathrm{~s}, 3 \mathrm{H}), 1.10(\mathrm{~d}$, $J=7.26 \mathrm{~Hz}, 3 \mathrm{H}) ;{ }^{13} \mathrm{C}$ NMR $\left(75 \mathrm{MHz}, \mathrm{CDCl}_{3}\right) \delta(\mathrm{ppm}): 167.8,137.0,135.8,83.2,60.4$, 29.7, 24.9, 24.6, 15.1, 14.4, 14.3; ${ }^{11}$ B NMR $\delta$ (ppm): 33; EI-MS: m/z $268(\mathrm{M})^{+}, 96$; CIMS: $\mathrm{m} / \mathrm{z} 269(\mathrm{M}+\mathrm{H})^{+}, 169$; HRMS-EI: 268.1846 (calcd.), 268.1842 (actual).

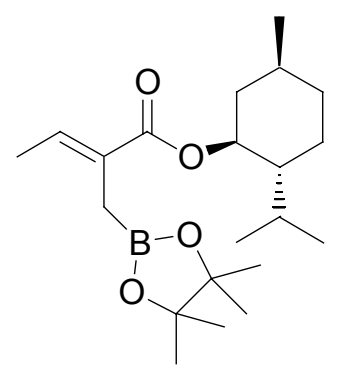

9d.

${ }^{1} \mathrm{H}$ NMR $\left(300 \mathrm{MHz}, \mathrm{CDCl}_{3}\right) \delta(\mathrm{ppm}): 6.77(\mathrm{q}, J=6.93 \mathrm{~Hz}, 1 \mathrm{H}), 4.71(\mathrm{dt}, J=10.59 \mathrm{~Hz}, J$ $=4.16 \mathrm{~Hz}, 1 \mathrm{H}), 1.95(\mathrm{~m}, 2 \mathrm{H}), 1.76(\mathrm{~d}, J=6.99 \mathrm{~Hz}, 3 \mathrm{H}), 1.41(\mathrm{~m}, 2 \mathrm{H}), 1.22(\mathrm{~s}, 12 \mathrm{H}), 1.01$ (m, 3H), $0.87(\mathrm{~d}, J=6.09 \mathrm{~Hz}), 0.74(\mathrm{~d}, J=6.84 \mathrm{~Hz}, 3 \mathrm{H}) ;{ }^{13} \mathrm{C} \mathrm{NMR}\left(75 \mathrm{MHz}, \mathrm{CDCl}_{3}\right) \delta$ (ppm): 167.7, 134.7, 130.8, 83.2, 74.1, 47.2, 41.0, 34.4, 31.4, 29.7, 26.2, 24.8, 23.4, 22.1, 


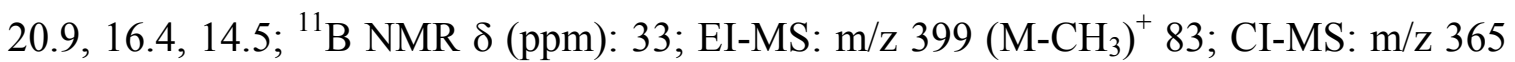
$(\mathrm{M}+\mathrm{H})^{+}, 209$ [(M+H-Menthol) ${ }^{+}, 100 \%$ ]; HRMS-CI: 365.2863 (calcd.), 365.2863 (actual).

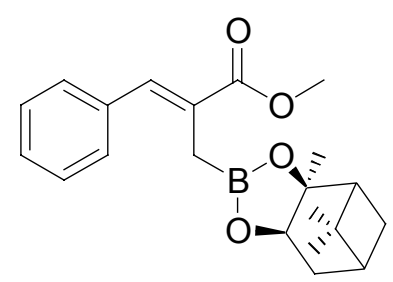

10a.

${ }^{1} \mathrm{H}$ NMR $\left(300 \mathrm{MHz}, \mathrm{CDCl}_{3}\right) \delta$ (ppm): $7.69(\mathrm{~s}, 1 \mathrm{H}), 7.42-7.29$ (m, 5H), $4.29(\mathrm{dd}, J=1.98$ $\mathrm{Hz}, J=2.04 \mathrm{~Hz}), 3.81(\mathrm{~s}, 3 \mathrm{H}), 2.32(\mathrm{~m}, 1 \mathrm{H}), 2.19(\mathrm{~m}, 2 \mathrm{H}), 2.05(\mathrm{t}, J=5.64 \mathrm{~Hz}, 1 \mathrm{H}), 1.90$ $(\mathrm{m}, 1 \mathrm{H}), 1.82(\mathrm{~m}, 1 \mathrm{H}), 1.37(\mathrm{~s}, 3 \mathrm{H}), 1.28(\mathrm{~s}, 3 \mathrm{H}), 1.21(\mathrm{~d}, J=10.71 \mathrm{~Hz}), 0.84(\mathrm{~s}, 3 \mathrm{H}) ;{ }^{13} \mathrm{C}$ NMR $\left(75 \mathrm{MHz}, \mathrm{CDCl}_{3}\right) \delta(\mathrm{ppm}): 169.1,137.6,136.2,130.2,129.4,128.4,128.1,85.9$, 78.0, 52.1, 51.3, 39.5, 38.2, 35.5, 28.6, 27.1, 26.3, 24.1; ${ }^{11} \mathrm{~B}$ NMR $\delta$ (ppm): 32; EI-MS: $\mathrm{m} / \mathrm{z} 354(\mathrm{M})^{+}, 116$; CI-MS: m/z $365(\mathrm{M}+\mathrm{H})^{+}, 203\left[\left(\mathrm{C}_{6} \mathrm{H}_{5} \mathrm{CHC}\left(\mathrm{CH}_{2} \mathrm{BOH}\right) \mathrm{CO}_{2} \mathrm{H}\right)^{+}\right.$, 100\%]; HRMS-EI: 354.2002 (calcd.), 354.2006 (actual).

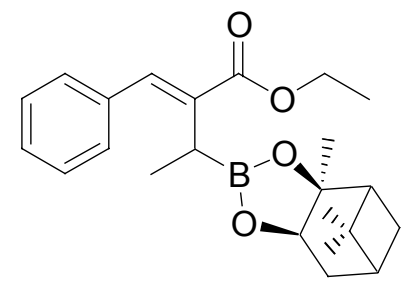

10c.

${ }^{1} \mathrm{H}$ NMR (300 MHz, $\left.\mathrm{CDCl}_{3}\right) \delta(\mathrm{ppm})$ : $7.71(\mathrm{~s}, 1 \mathrm{H}), 7.49-7.32(\mathrm{~m}, 5 \mathrm{H}), 4.35(\mathrm{~m}, 3 \mathrm{H}), 2.63$ $(\mathrm{m}, 1 \mathrm{H}), 2.38(\mathrm{~m}, 1 \mathrm{H}), 2.24(\mathrm{~m}, 1 \mathrm{H}), 2.10(\mathrm{q}, J=5.9 \mathrm{~Hz}, 1 \mathrm{H}), 1.94(\mathrm{~m}, 2 \mathrm{H}), 1.48(\mathrm{~s}, 1 \mathrm{H})$, $1.37(\mathrm{~m}, 13 \mathrm{H}), 0.89(\mathrm{~s}, 3 \mathrm{H}) ;{ }^{13} \mathrm{C}$ NMR $\left(75 \mathrm{MHz}, \mathrm{CDCl}_{3}\right) \delta(\mathrm{ppm}): 167.3,136.4,135.2$, 128.2, 127.3, 127.0, 84.7, 76.9, 59.8, 50.3, 38.5, 37.2, 34.6, 28.7, 27.6, 27.4, 26.1, 25.3, 23.0, 14.6, 13.3; ${ }^{11}$ B NMR $\delta$ (ppm): 33; EI-MS: m/z $382(\mathrm{M})^{+}$; CI-MS: m/z $383(\mathrm{M}+\mathrm{H})^{+}$, $231\left[(\mathrm{M}+\mathrm{H})-\mathrm{C}_{9} \mathrm{H}_{14} \mathrm{O}_{2}\right]$.

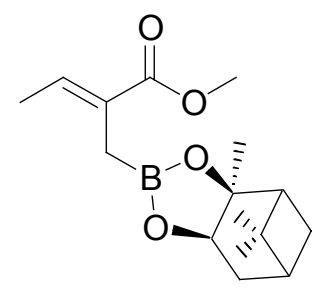

11a.

${ }^{1} \mathrm{H}$ NMR $\left(300 \mathrm{MHz}, \mathrm{CDCl}_{3}\right) \delta(\mathrm{ppm}): 6.81(\mathrm{q}, J=7.03 \mathrm{~Hz}, 1 \mathrm{H}), 4.24(\mathrm{~s}, \mathrm{~J}=8.58 \mathrm{~Hz}$, $1 \mathrm{H}), 3.69(\mathrm{~s}, 3 \mathrm{H}), 2.25(\mathrm{~m}, 1 \mathrm{H}), 2.14(\mathrm{~s}, 2 \mathrm{H}), 2.02(\mathrm{t}, J=5.59 \mathrm{~Hz}, 1 \mathrm{H}), 1.89(\mathrm{~s}, 3 \mathrm{H}), 1.81$ (m, 1H), 1.77 (d, $J=6.99 \mathrm{~Hz}, 3 \mathrm{H}), 1.35(\mathrm{~s}, 3 \mathrm{H}), 1.26(\mathrm{~s}, 3 \mathrm{H}), 1.19(\mathrm{~d}, J=10.71 \mathrm{~Hz}, 1 \mathrm{H})$, 
0,81 (s, 3H); ${ }^{13} \mathrm{C}$ NMR $\left(75 \mathrm{MHz}, \mathrm{CDCl}_{3}\right) \delta(\mathrm{ppm}): 168.5,135,7,130.1,85.7,77.8,51.6$, $51.2,39.5,38.2,35.5,28.6,27.1,26.3,24.0,14.5 ;{ }^{11} \mathrm{~B}$ NMR $\delta$ (ppm): 32; EI-MS: $\mathrm{m} / \mathrm{z}$ $292\left(\mathrm{M}^{+}\right.$, 54; CI-MS: m/z $293(\mathrm{M}+\mathrm{H})^{+}, 141$; HRMS-EI: 292.1846 (calcd.), 292.1840 (actual).

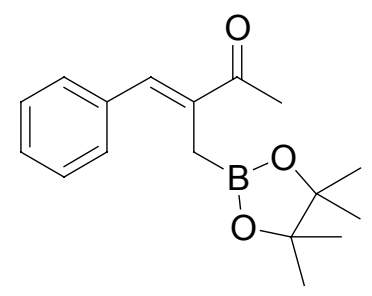

14.

${ }^{1} \mathrm{H}$ NMR $\left(300 \mathrm{MHz}, \mathrm{CDCl}_{3}\right) \delta(\mathrm{ppm}): 7.50(\mathrm{~s}, 1 \mathrm{H}), 7.44-7.30(\mathrm{~m}, 5 \mathrm{H}), 2.45(\mathrm{~s}, 3 \mathrm{H}), 2.01$ (s, 2H), 1.23 (s, $12 \mathrm{H}) ;{ }^{13} \mathrm{C}$ NMR (75 MHz, $\left.\mathrm{CDCl}_{3}\right) \delta(\mathrm{ppm}): 200.4,140.3,138.9,136.2$, 129.6, 129.3, 128.4, 83.2, 25.2, 24.8, 24.4; ${ }^{11}$ B NMR $\delta(\mathrm{ppm}): 33$; EI-MS: m/z $286(\mathrm{M})^{+}$, 142; CI-MS: m/z $287(\mathrm{M}+\mathrm{H})^{+}$; HRMS: 286.1740 (calcd.), 286.1737 (actual).

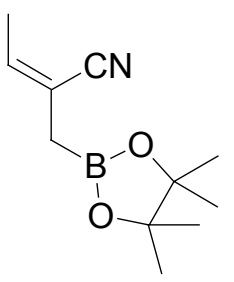

16.

${ }^{1} \mathrm{H}$ NMR (300 MHz, $\left.\mathrm{CDCl}_{3}\right) \delta(\mathrm{ppm}): 6.19(\mathrm{q}, J=6.91 \mathrm{~Hz}, 1 \mathrm{H}), 1.95(\mathrm{~d}, J=6.93 \mathrm{~Hz}$, $3 \mathrm{H}), 1.86(\mathrm{~s}, 2 \mathrm{H}), 1.26(\mathrm{~s}, 12 \mathrm{H}) ;{ }^{13} \mathrm{C}$ NMR $\left(75 \mathrm{MHz}, \mathrm{CDCl}_{3}\right) \delta$ (ppm): 142.3, 141.8, 84.0, 29.7, 24.7, 17.3; ${ }^{11} \mathrm{~B}$ NMR $\delta(\mathrm{ppm}): 32$; EI-MS: m/z $207(\mathrm{M})^{+}, 192\left(\mathrm{M}^{-} \mathrm{CH}_{3}\right), 85$ $\left(\mathrm{C}_{6} \mathrm{H}_{13}\right)^{+}$; CI-MS: m/z $208(\mathrm{M}+\mathrm{H})^{+}$. 


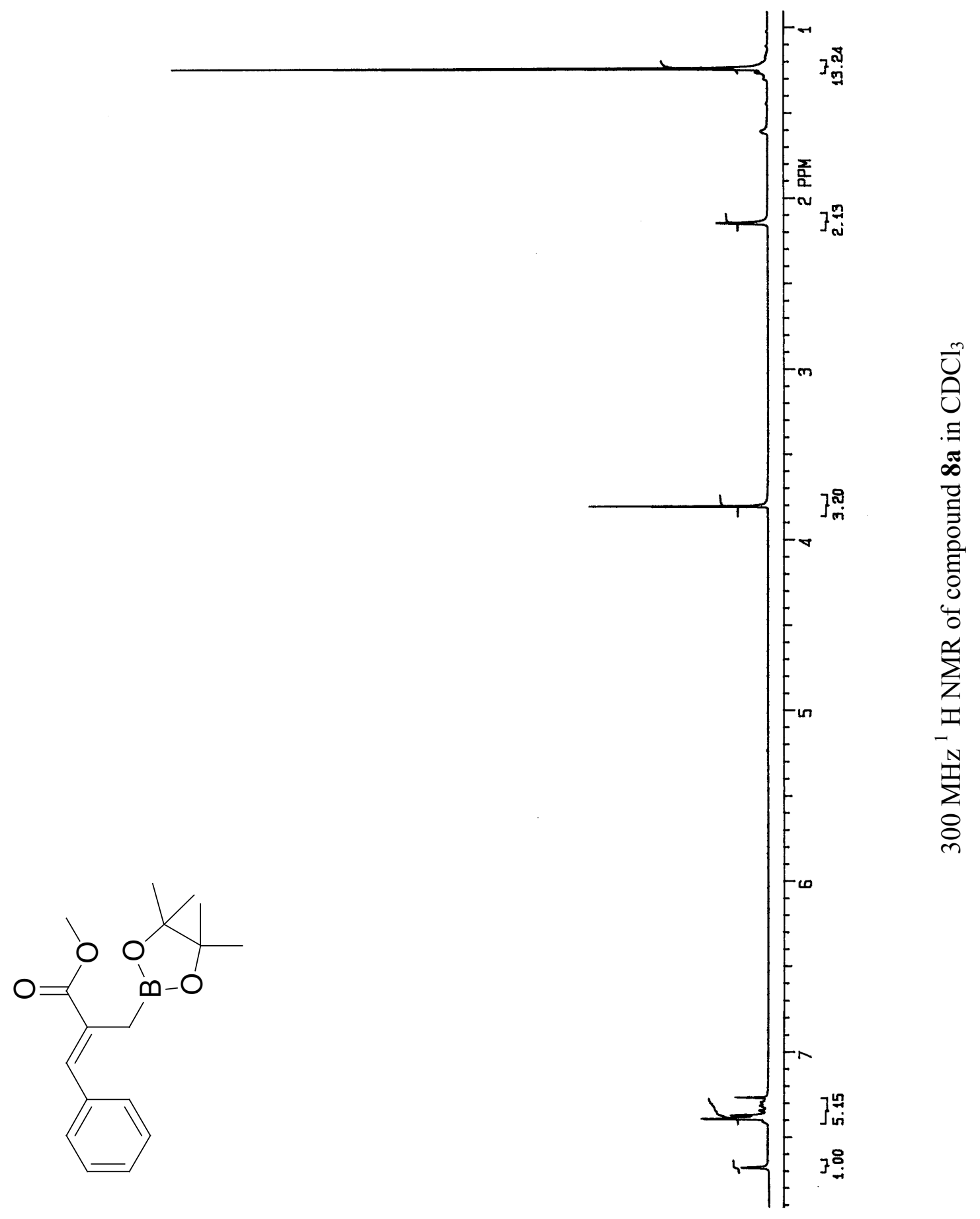




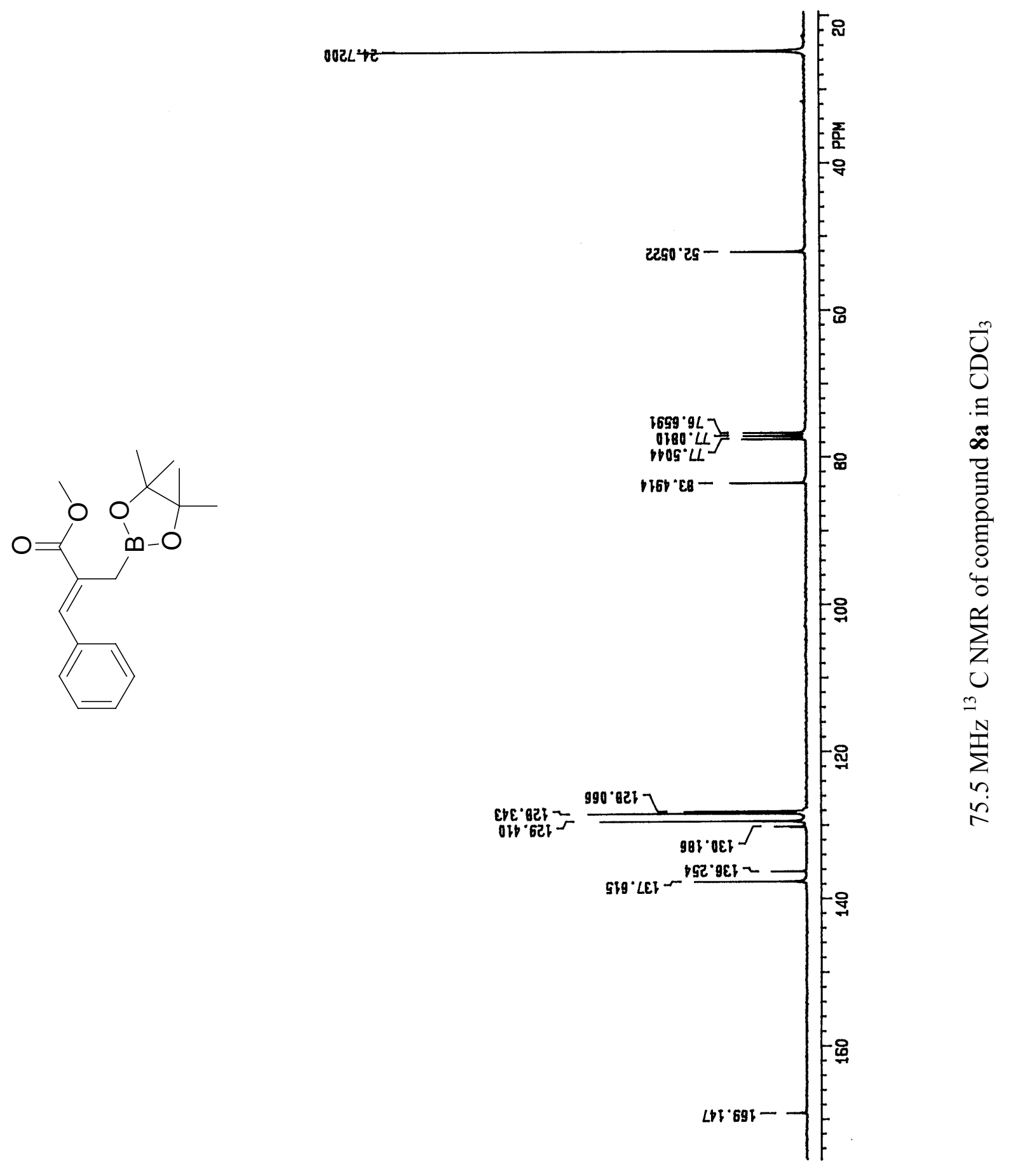



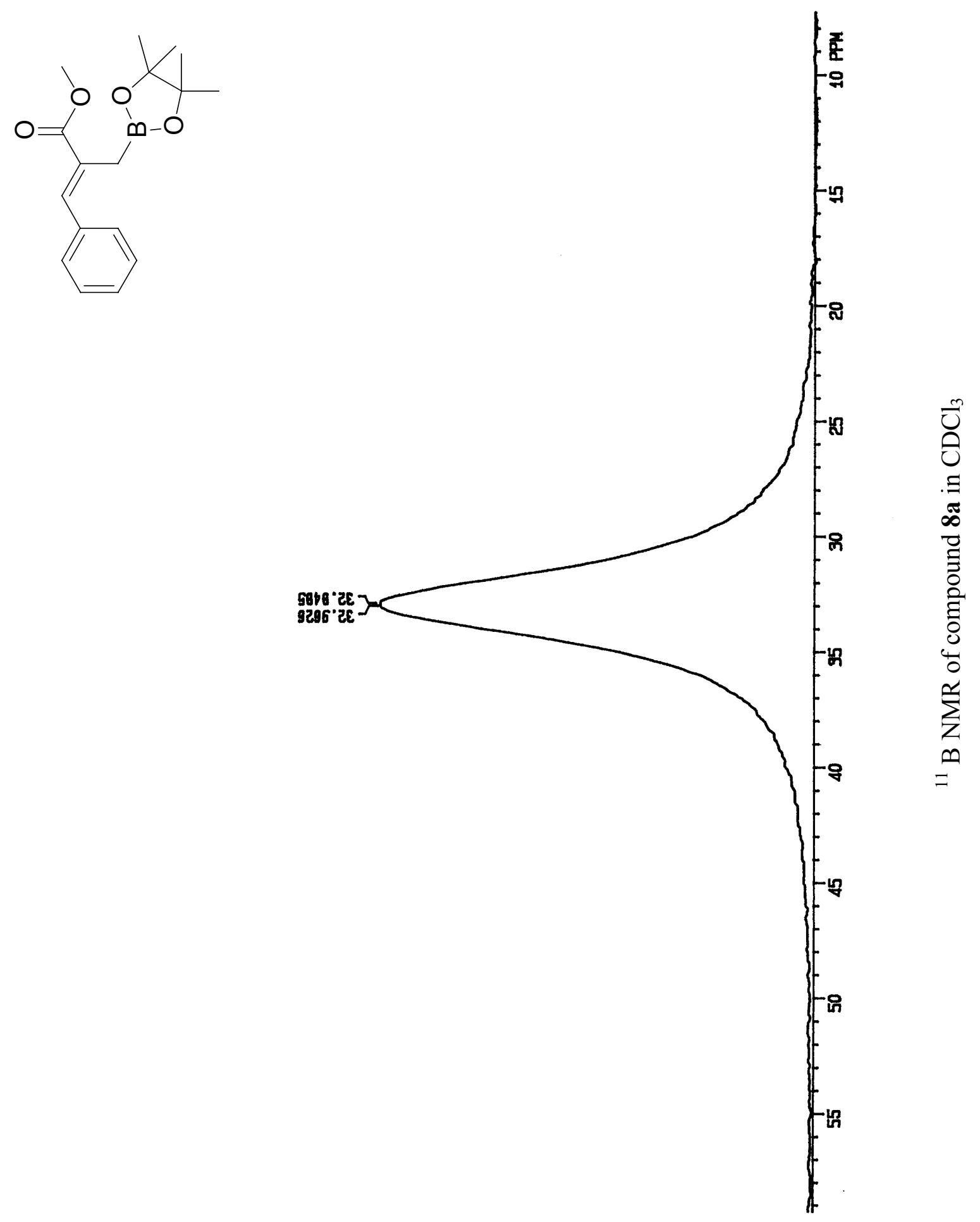


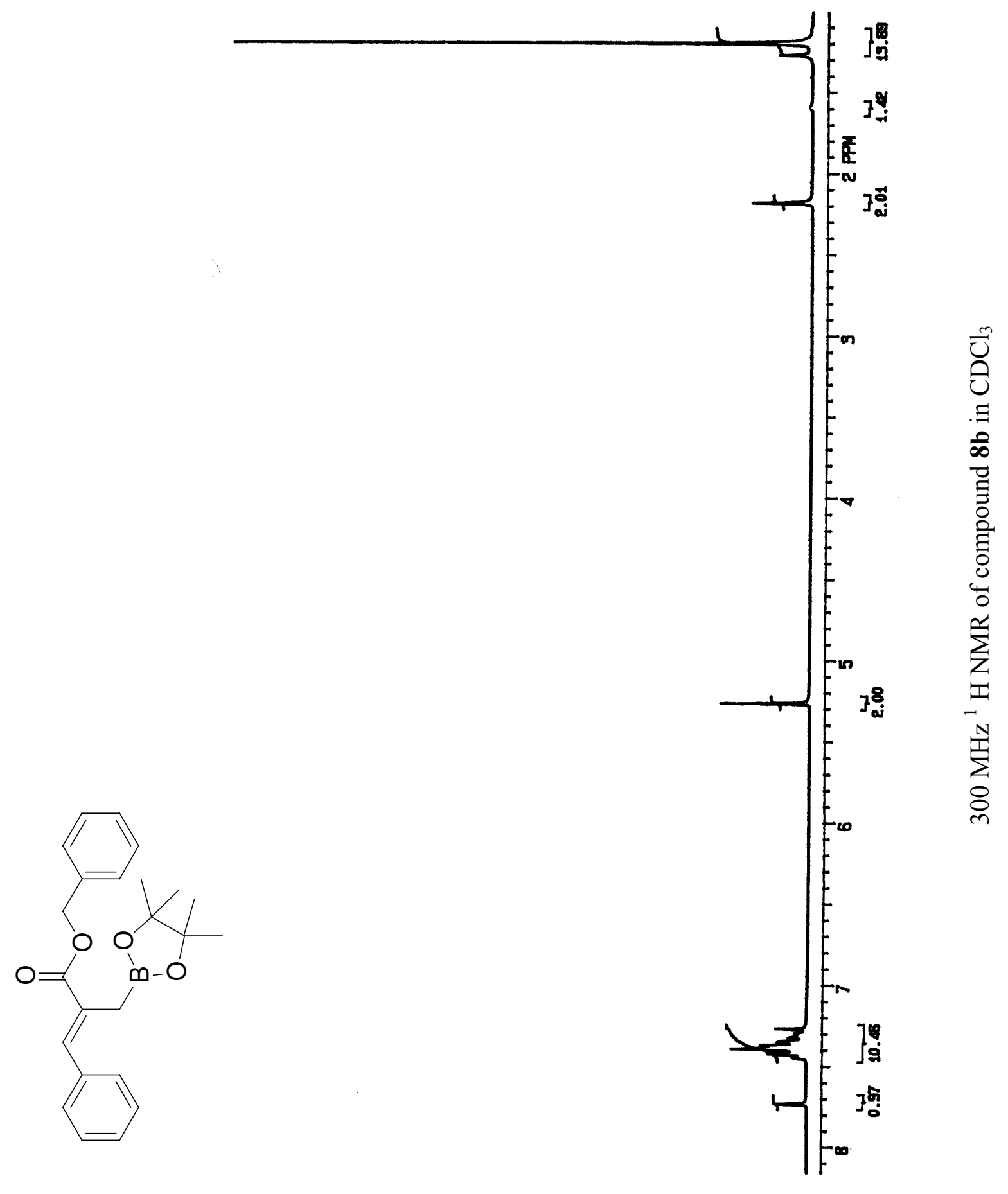



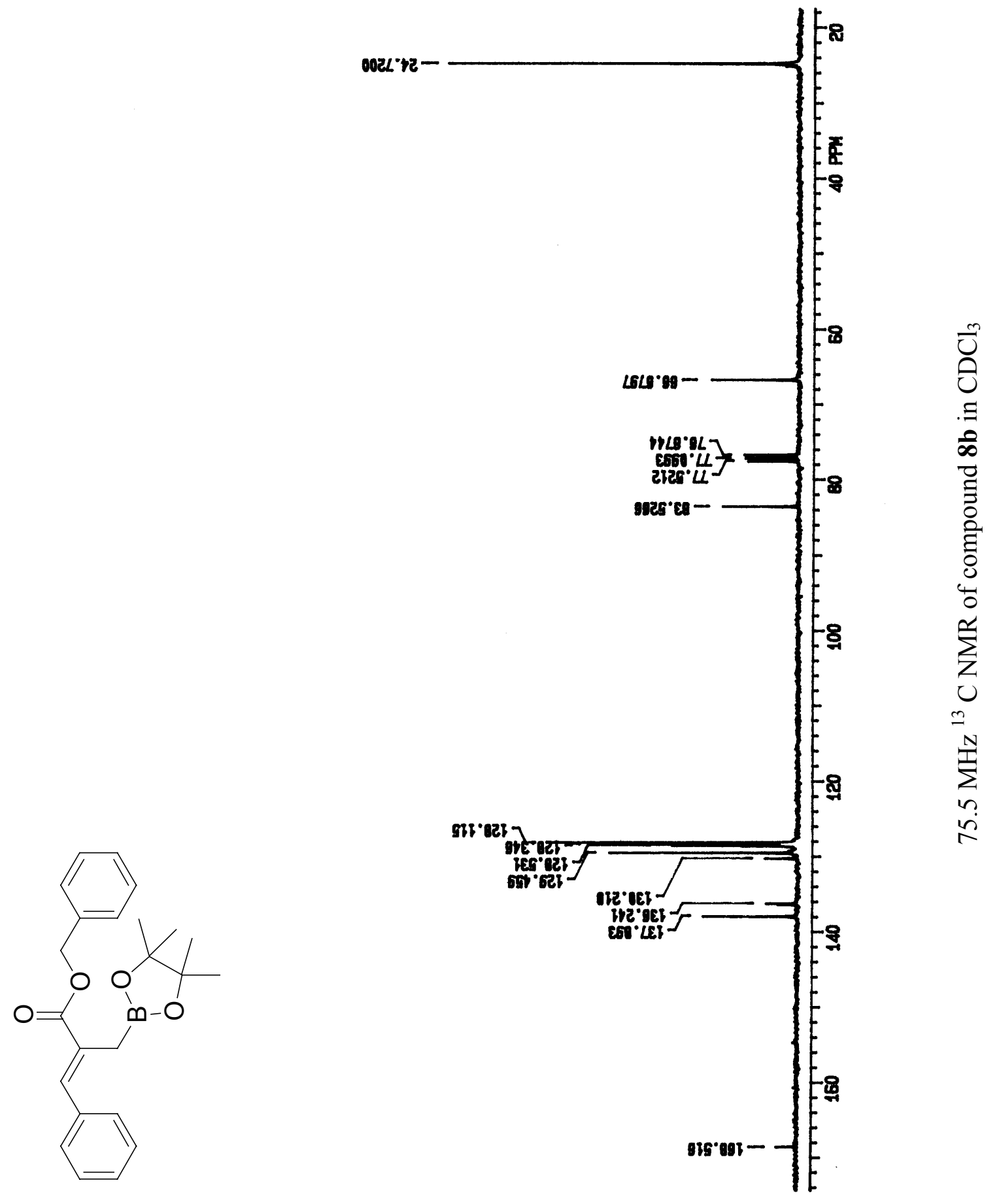

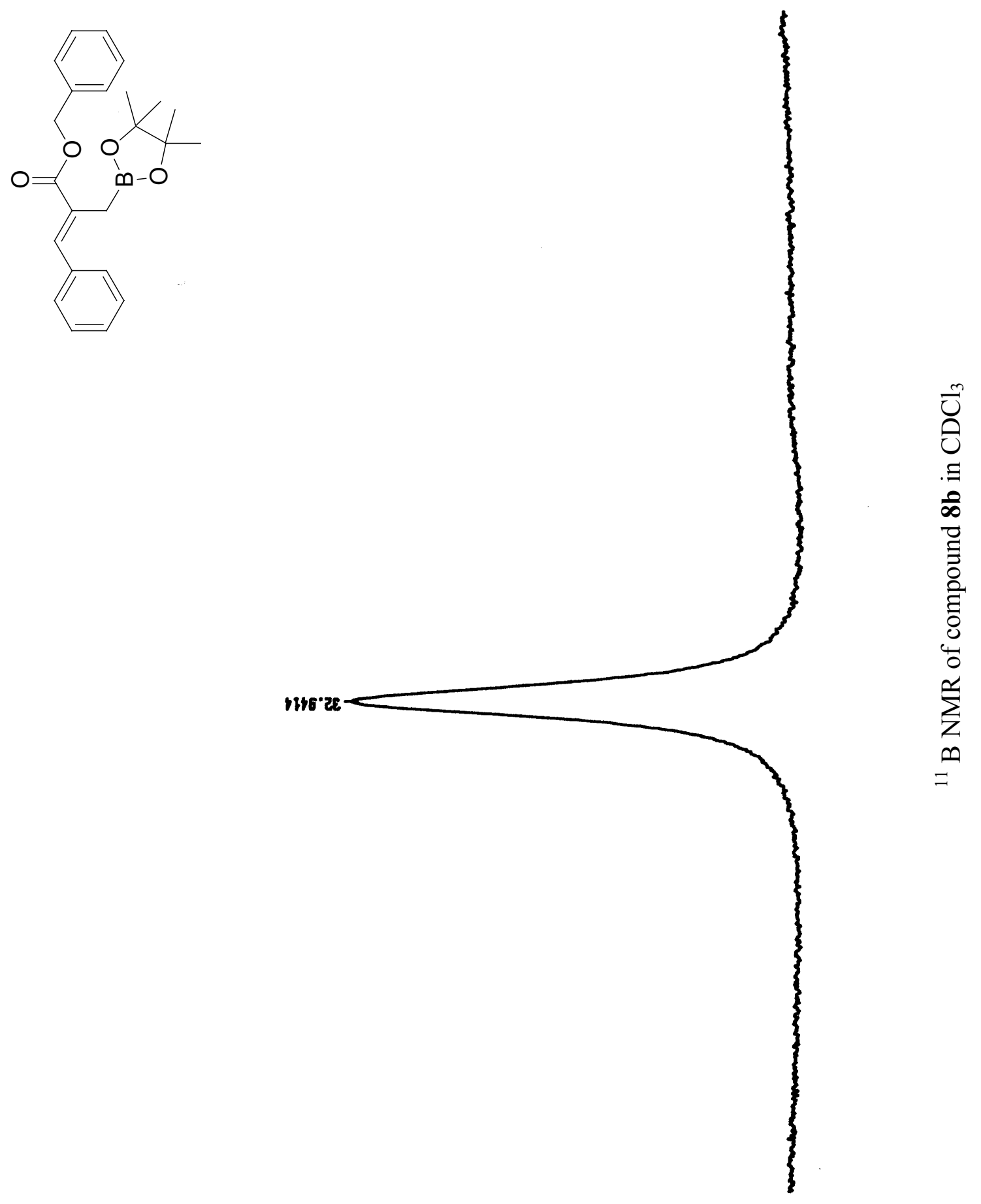


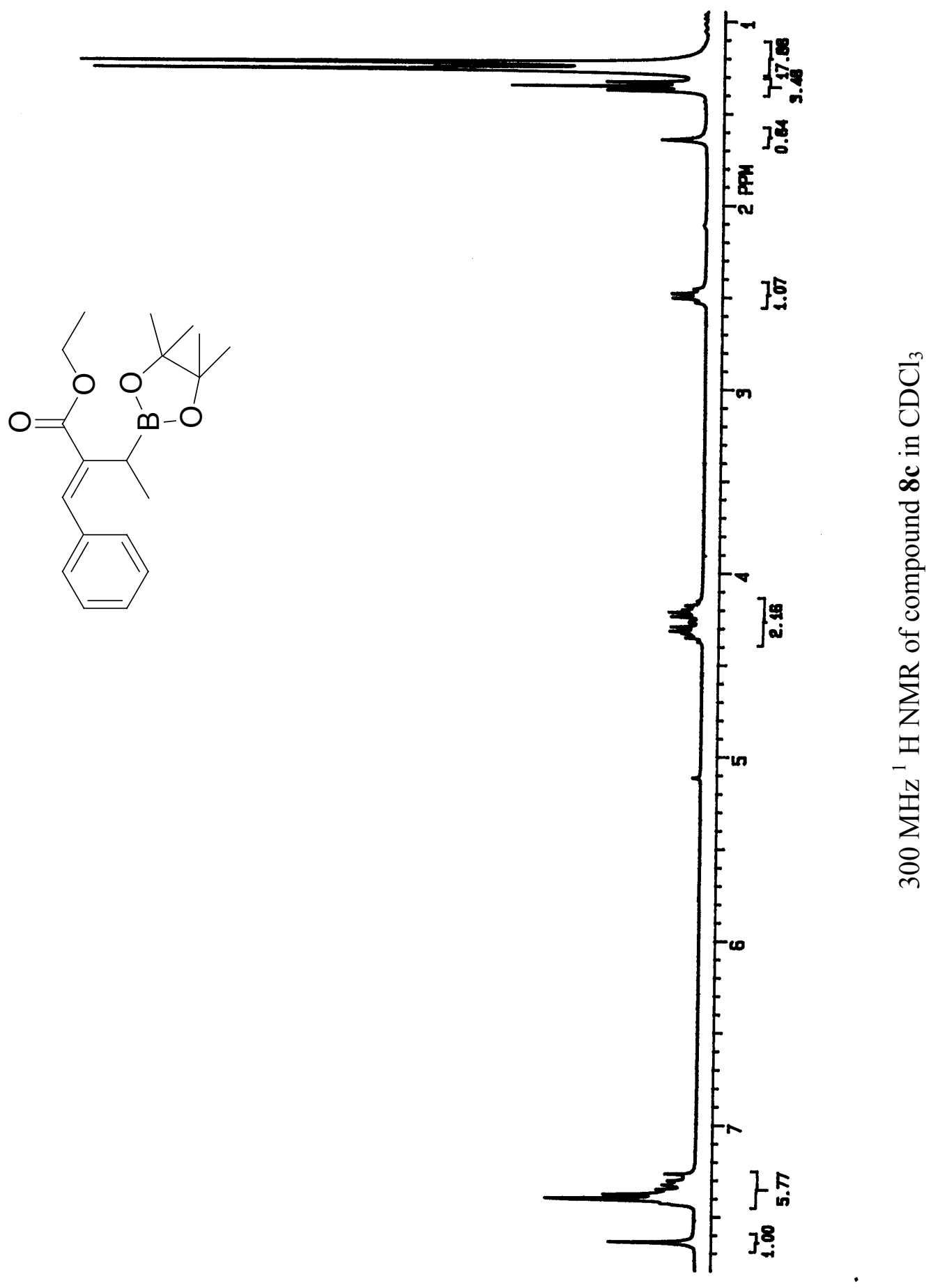



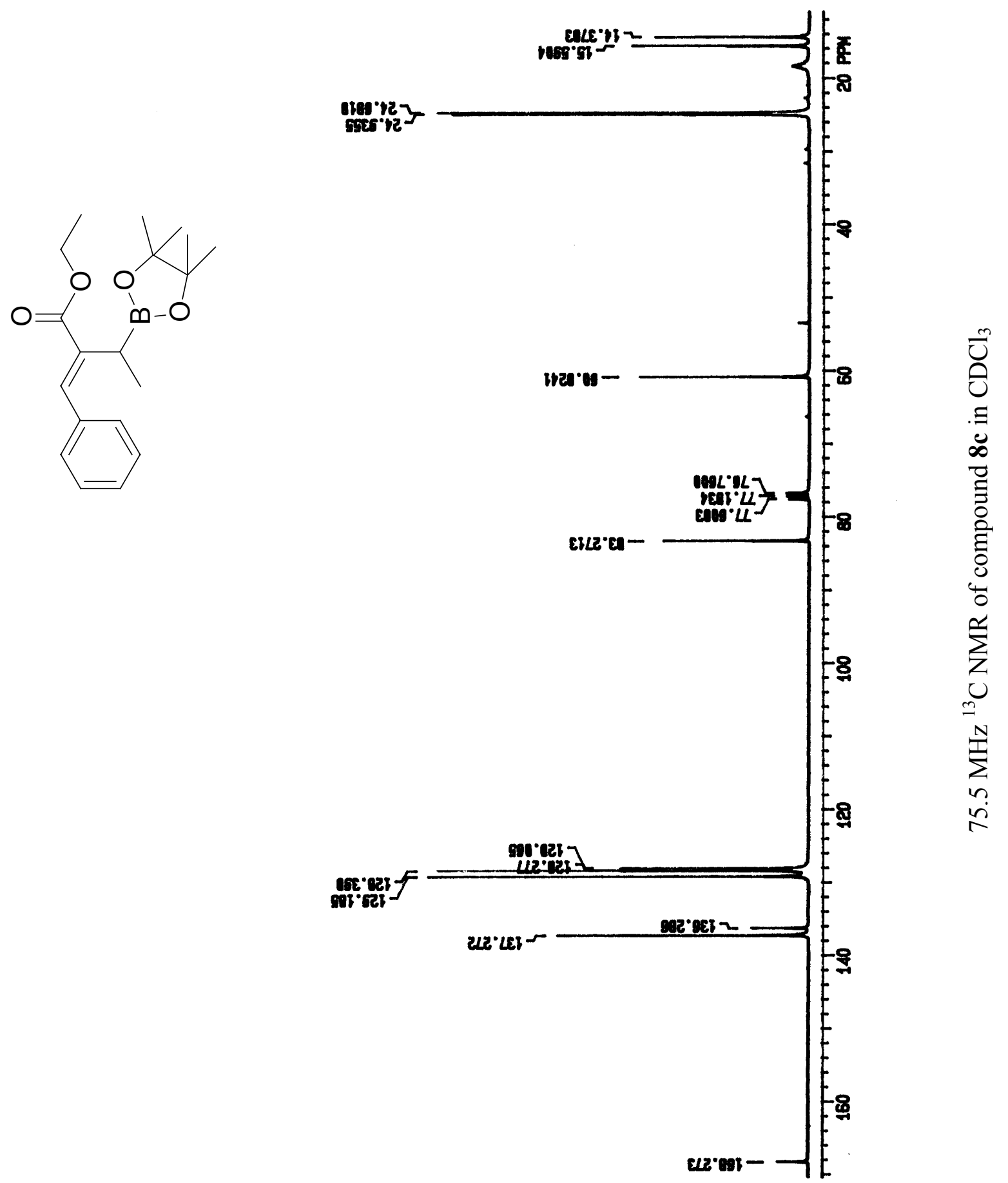

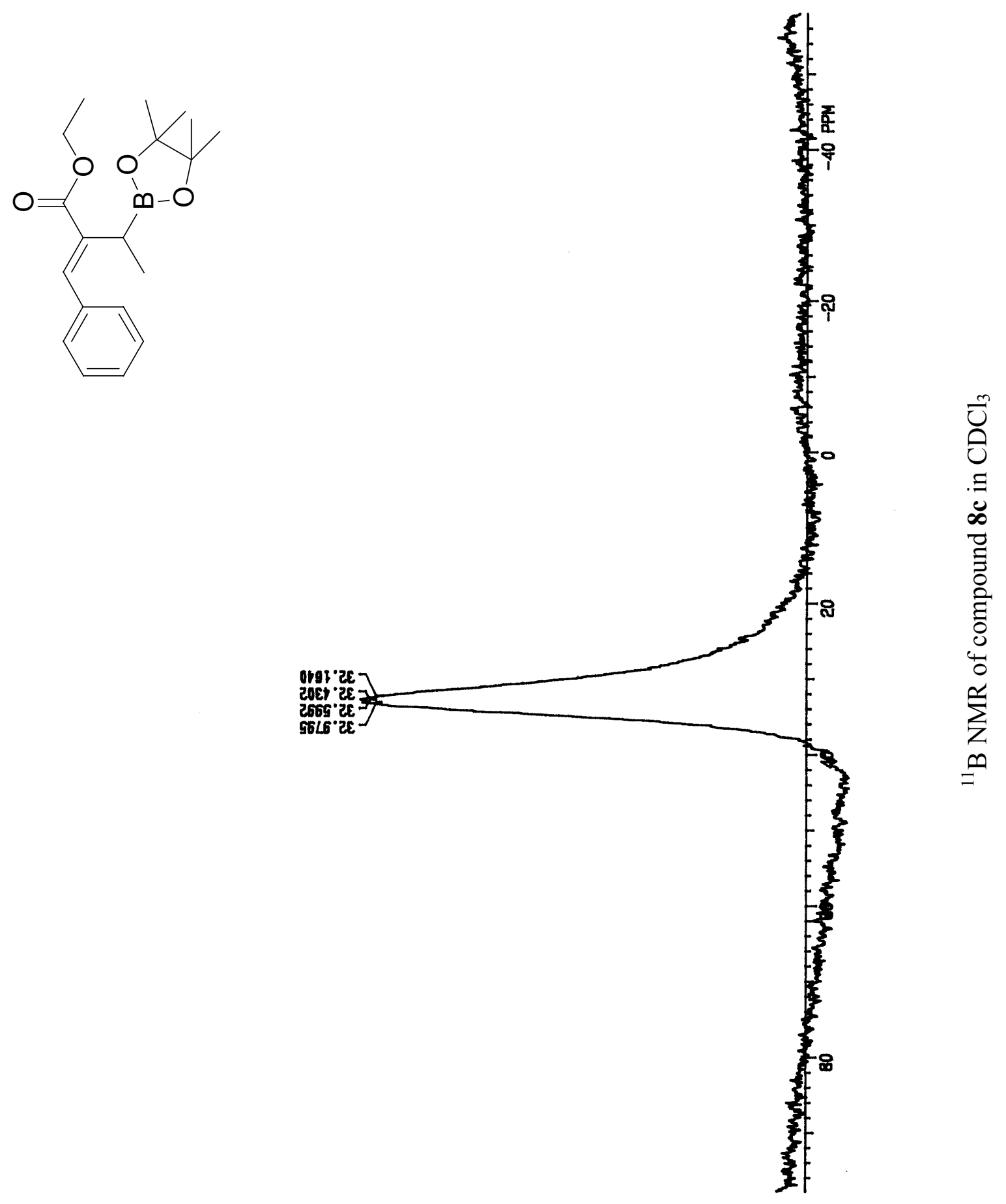


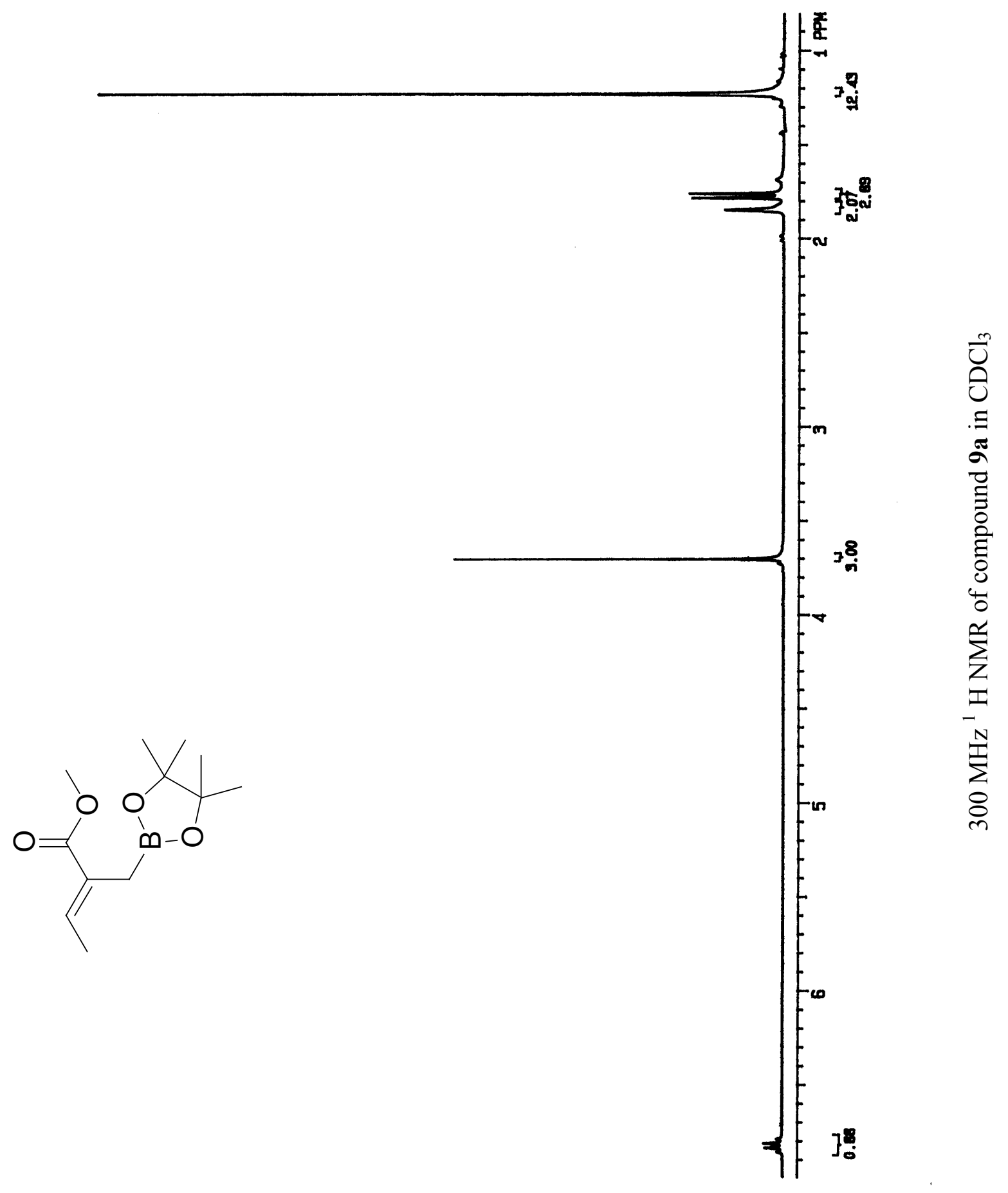




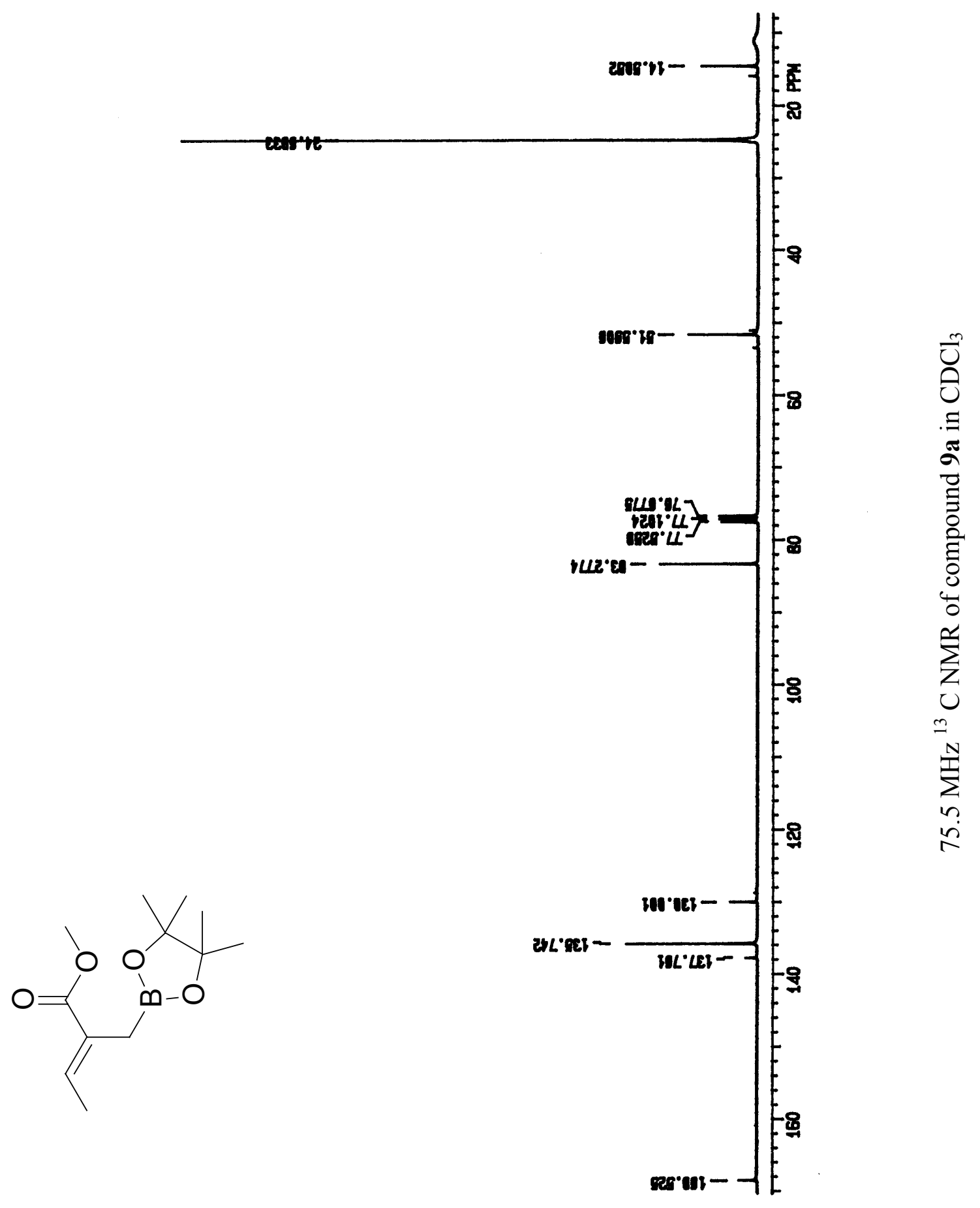




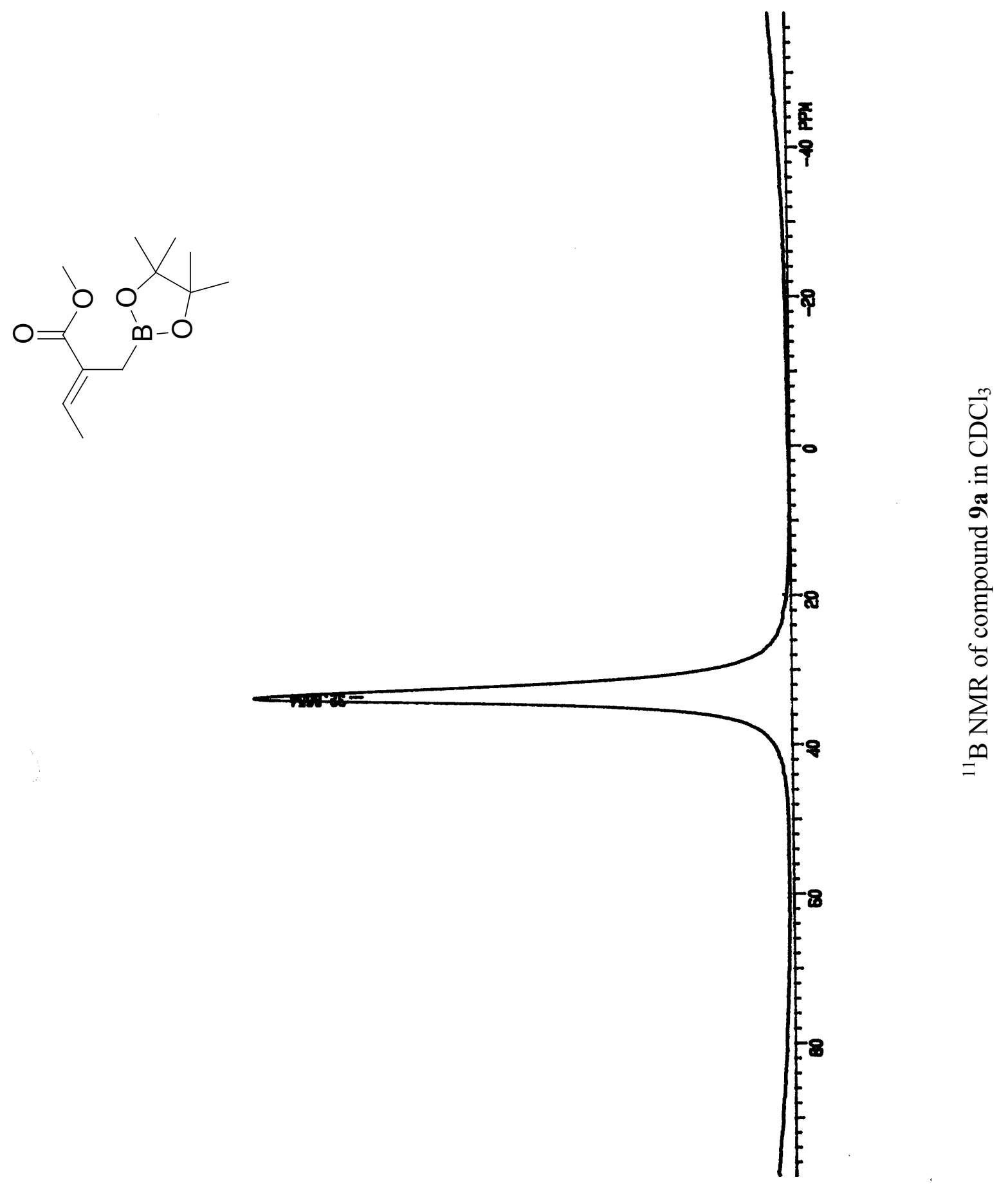




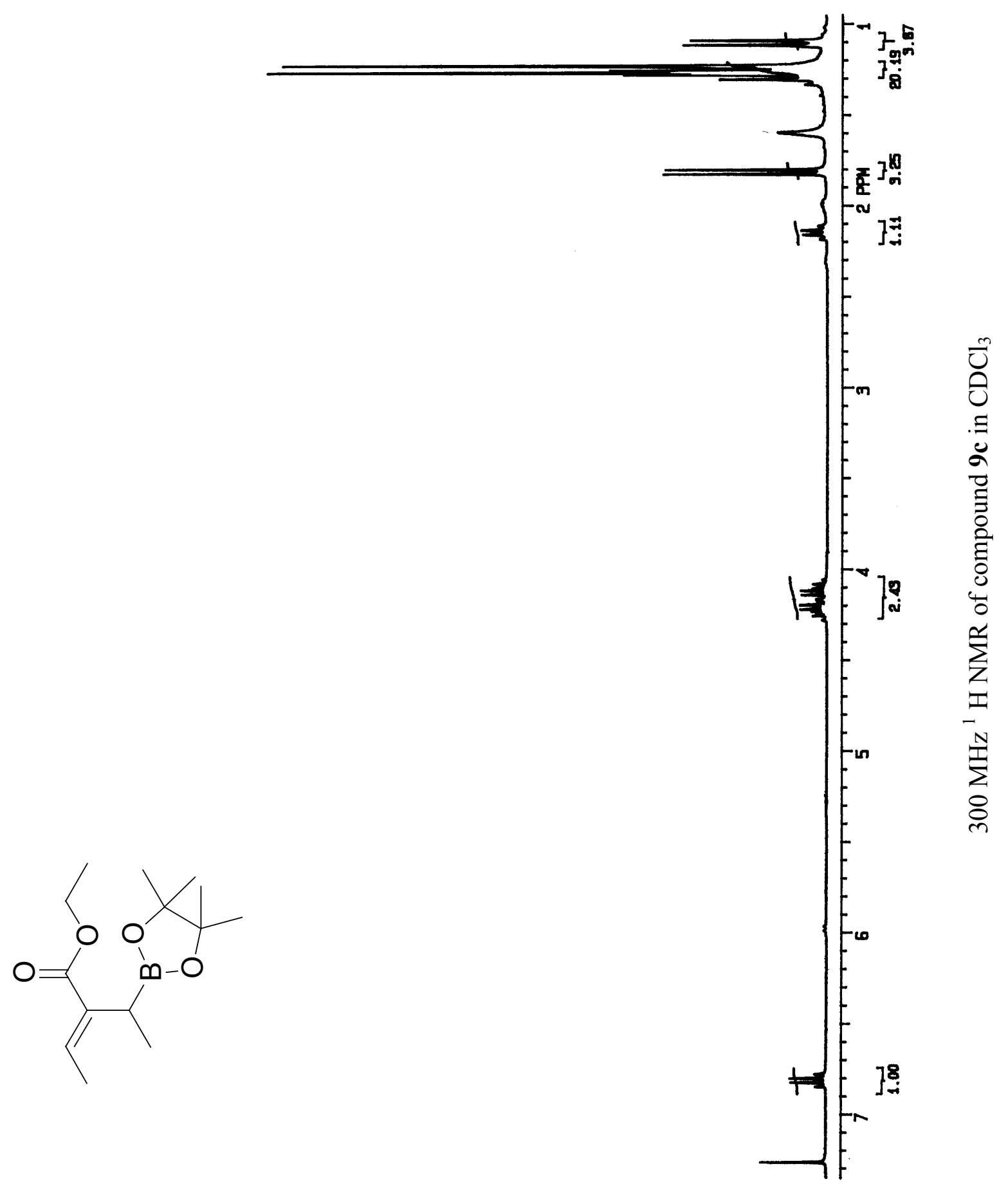




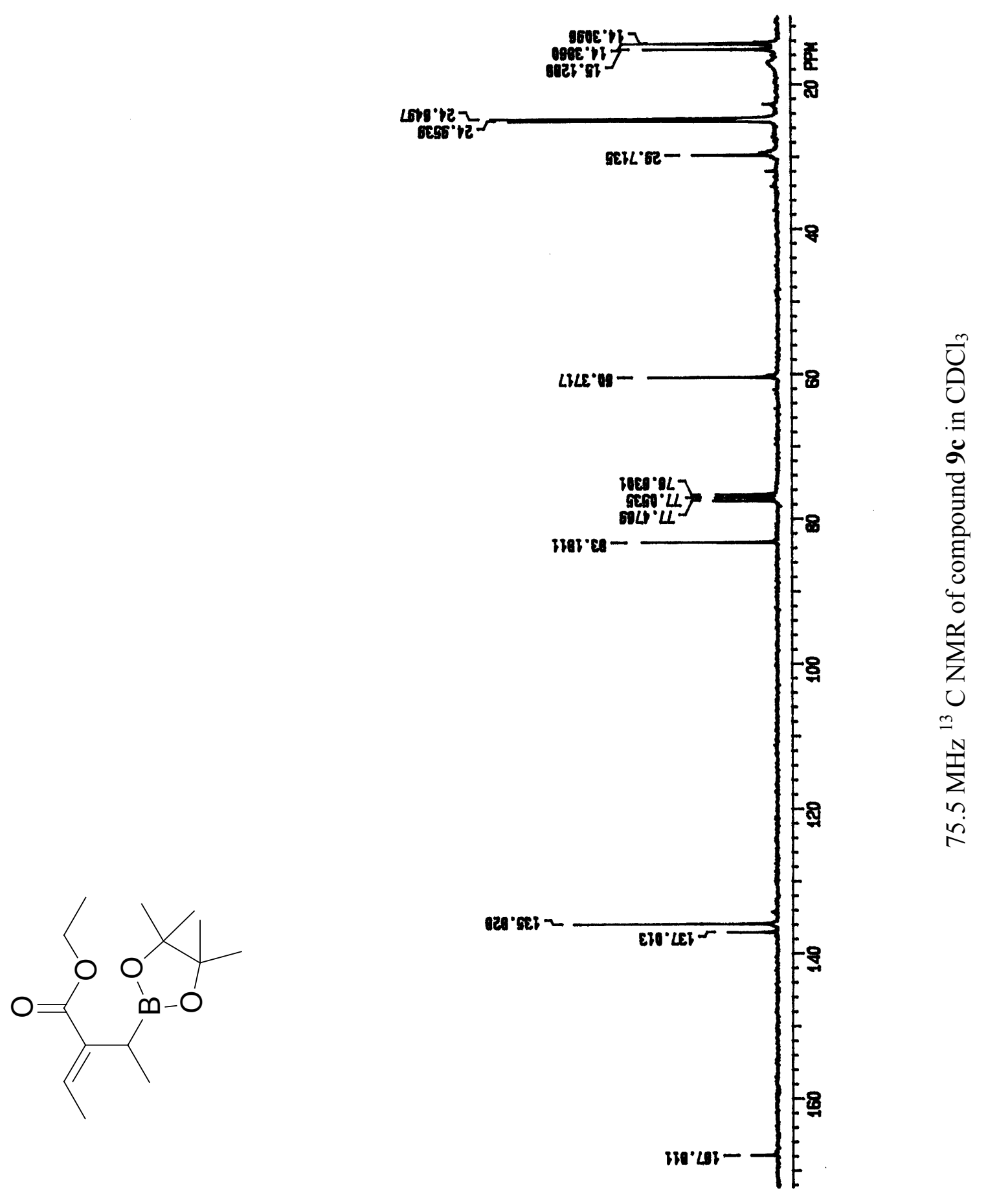




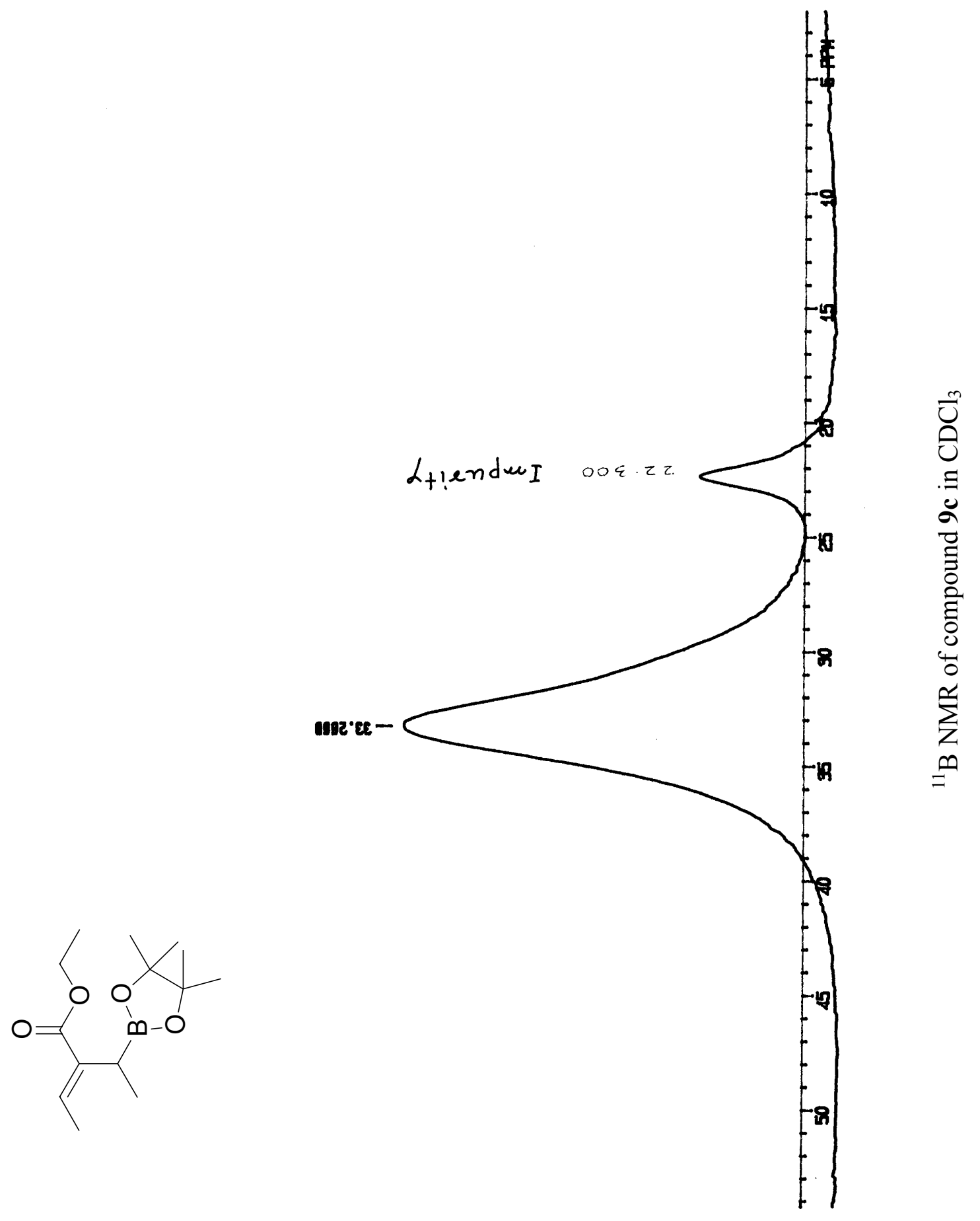




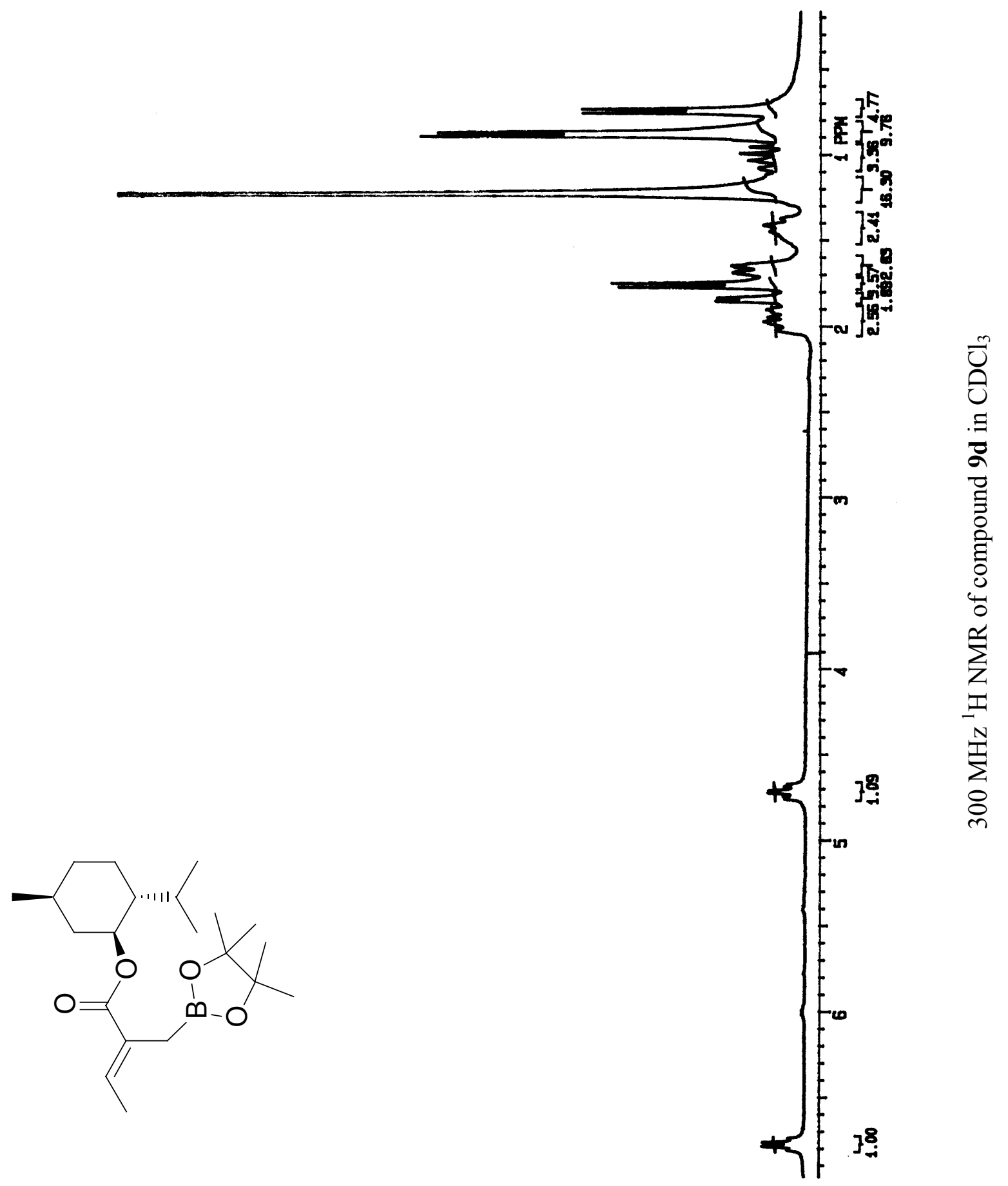




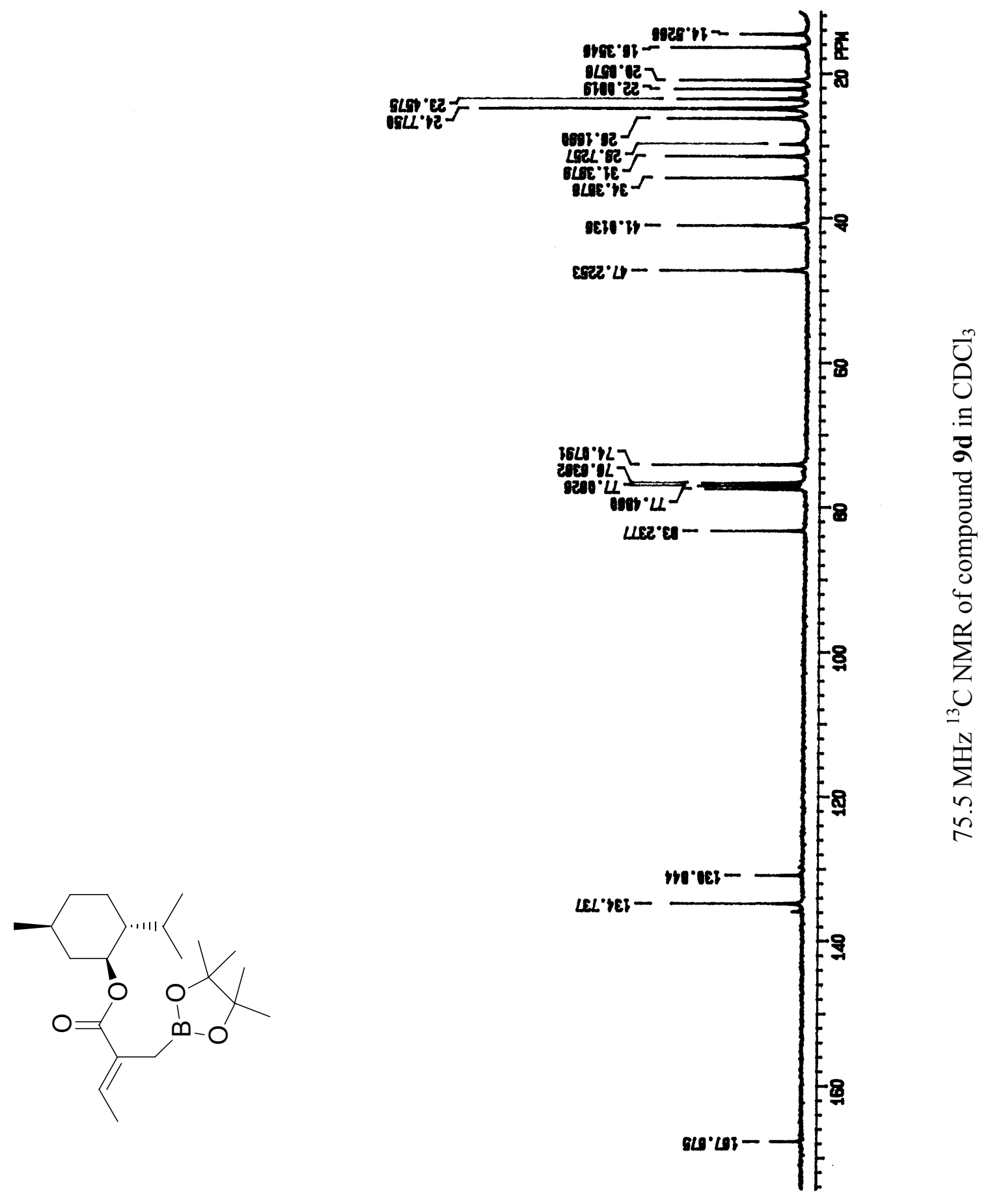




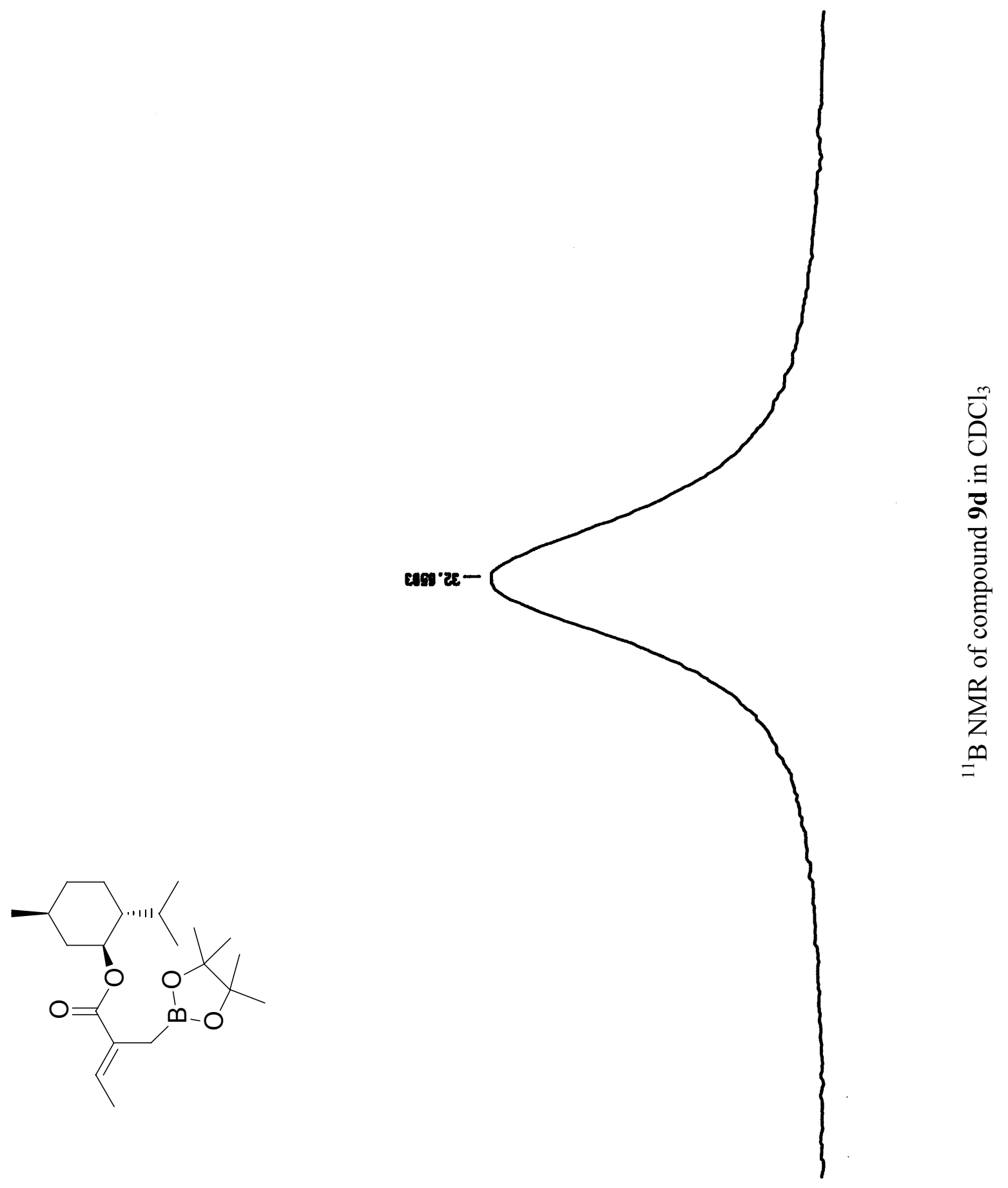




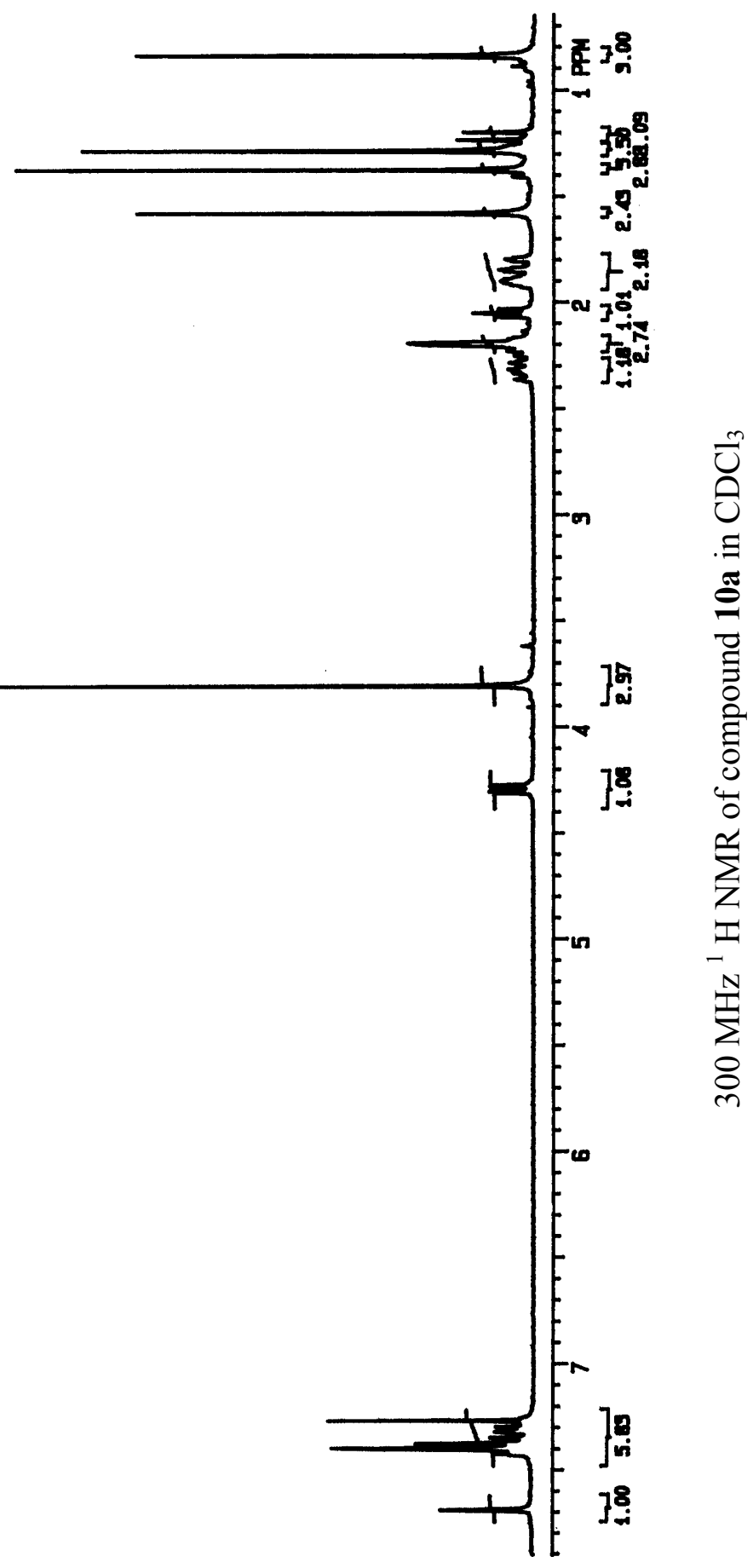



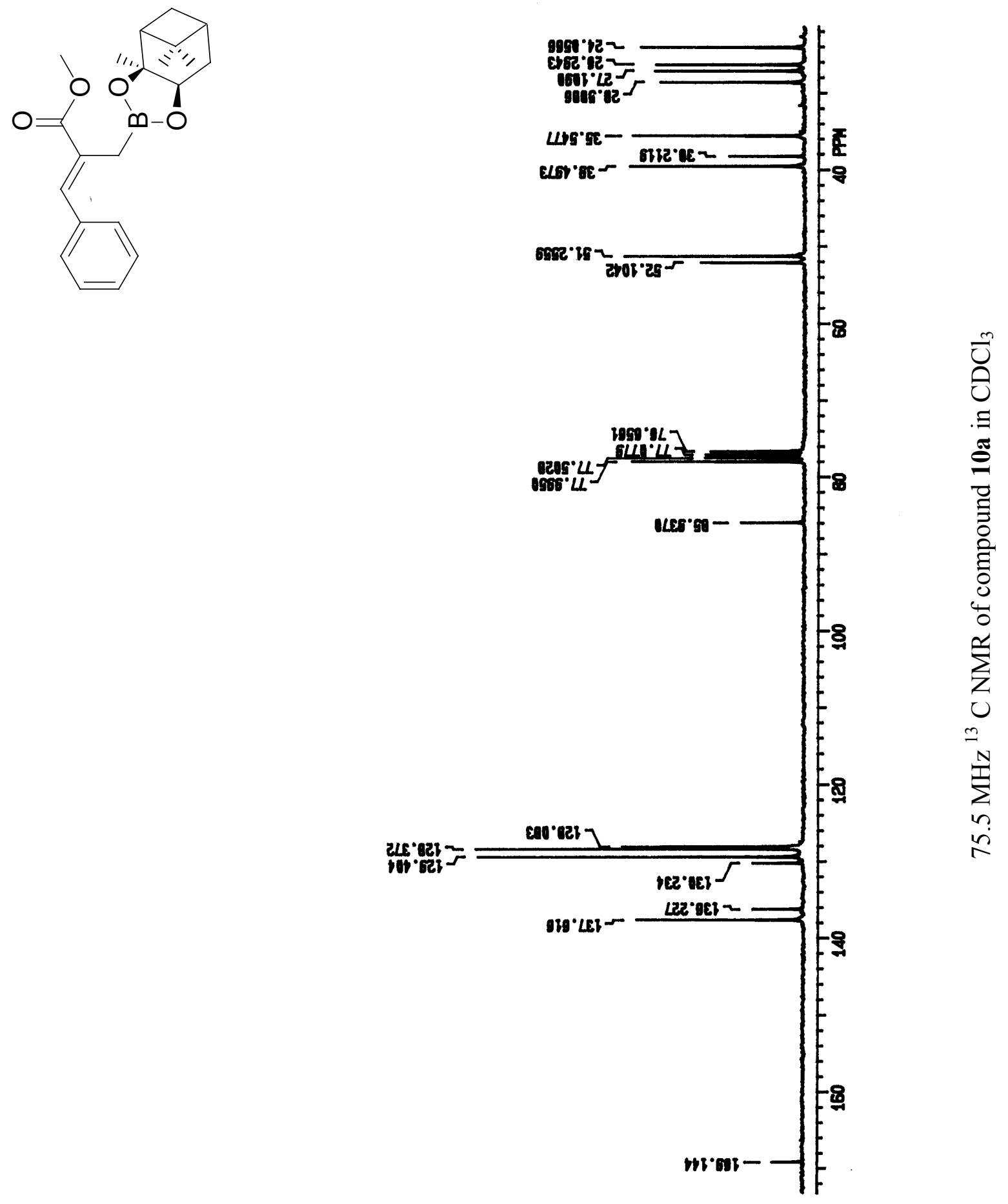

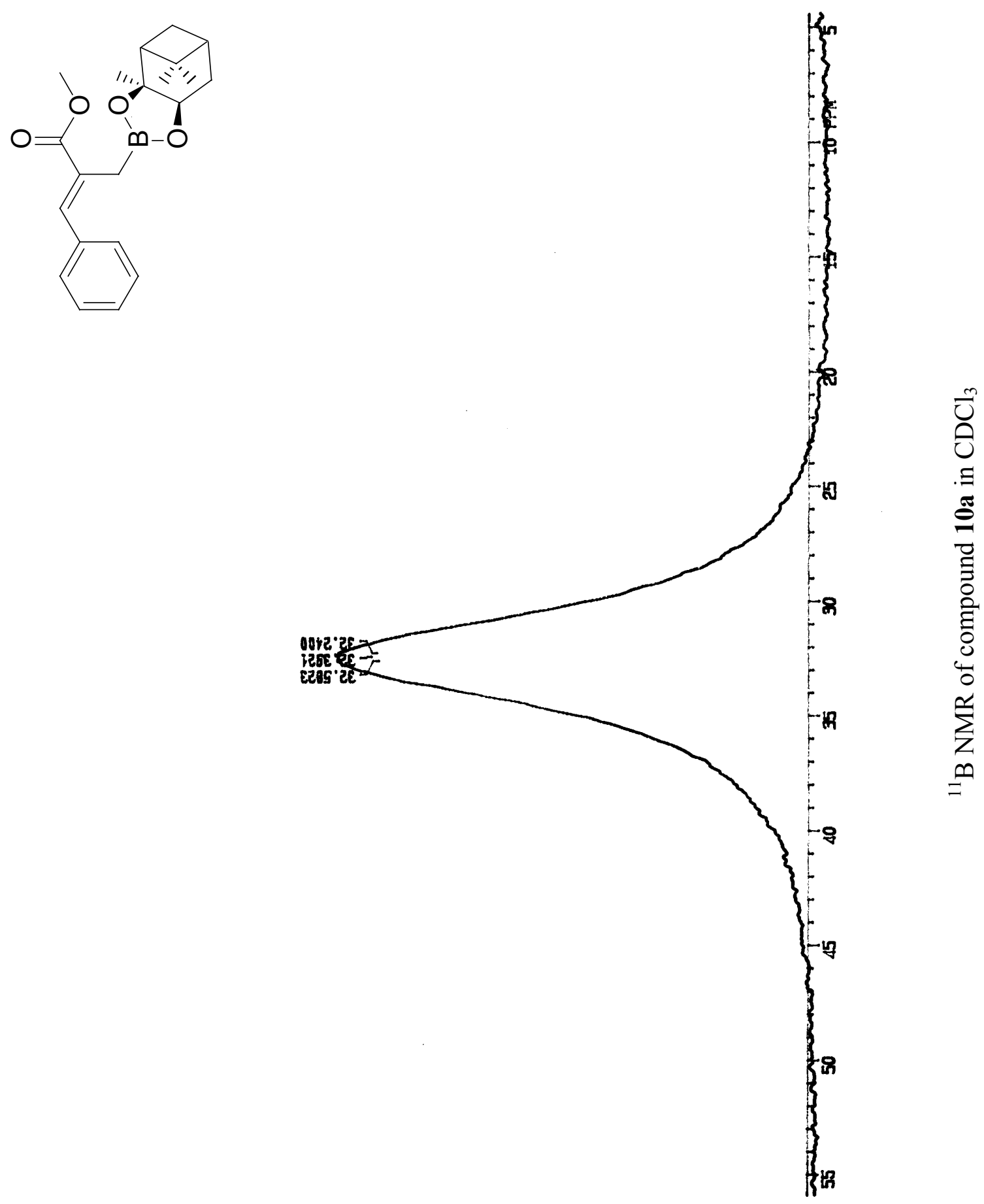


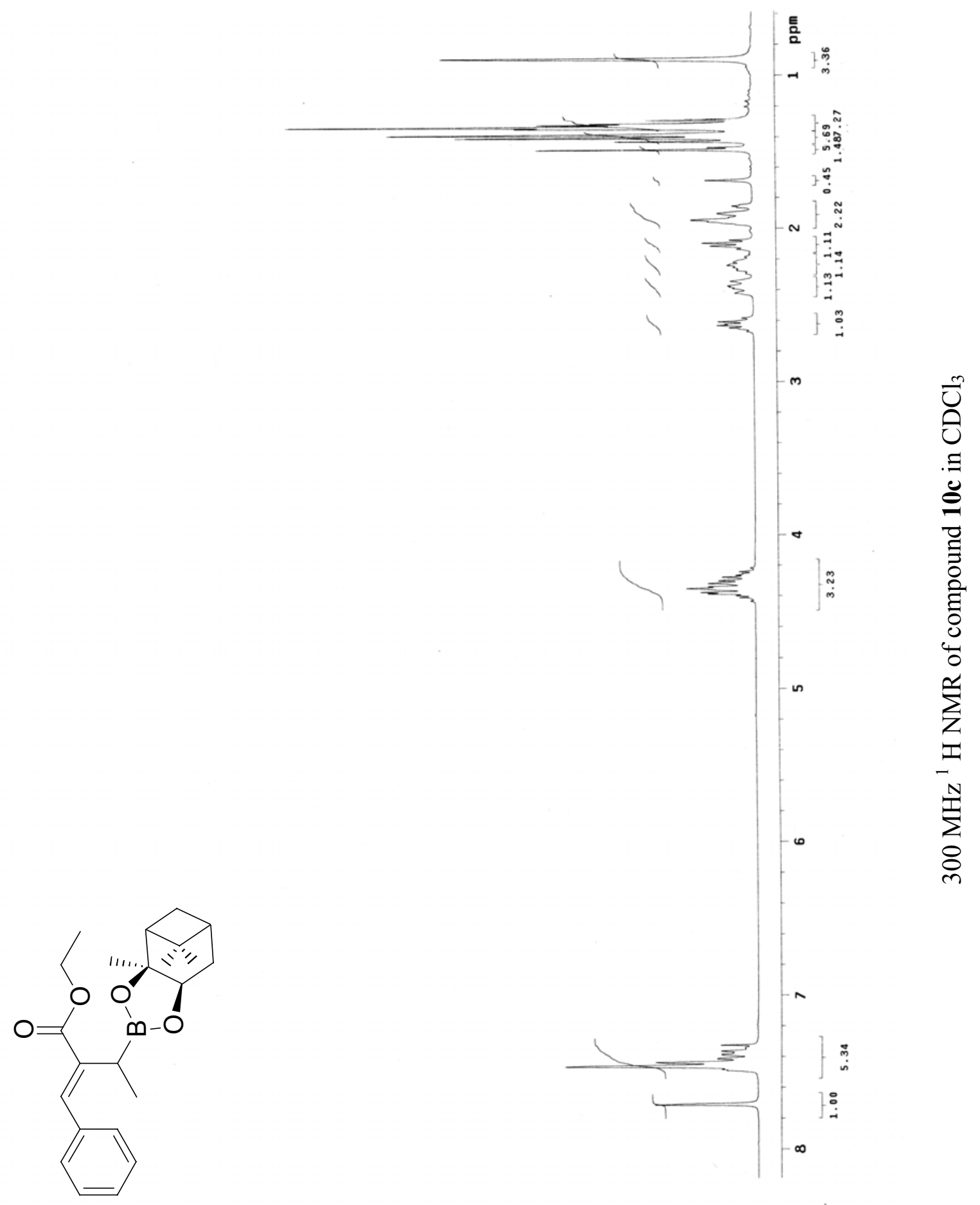



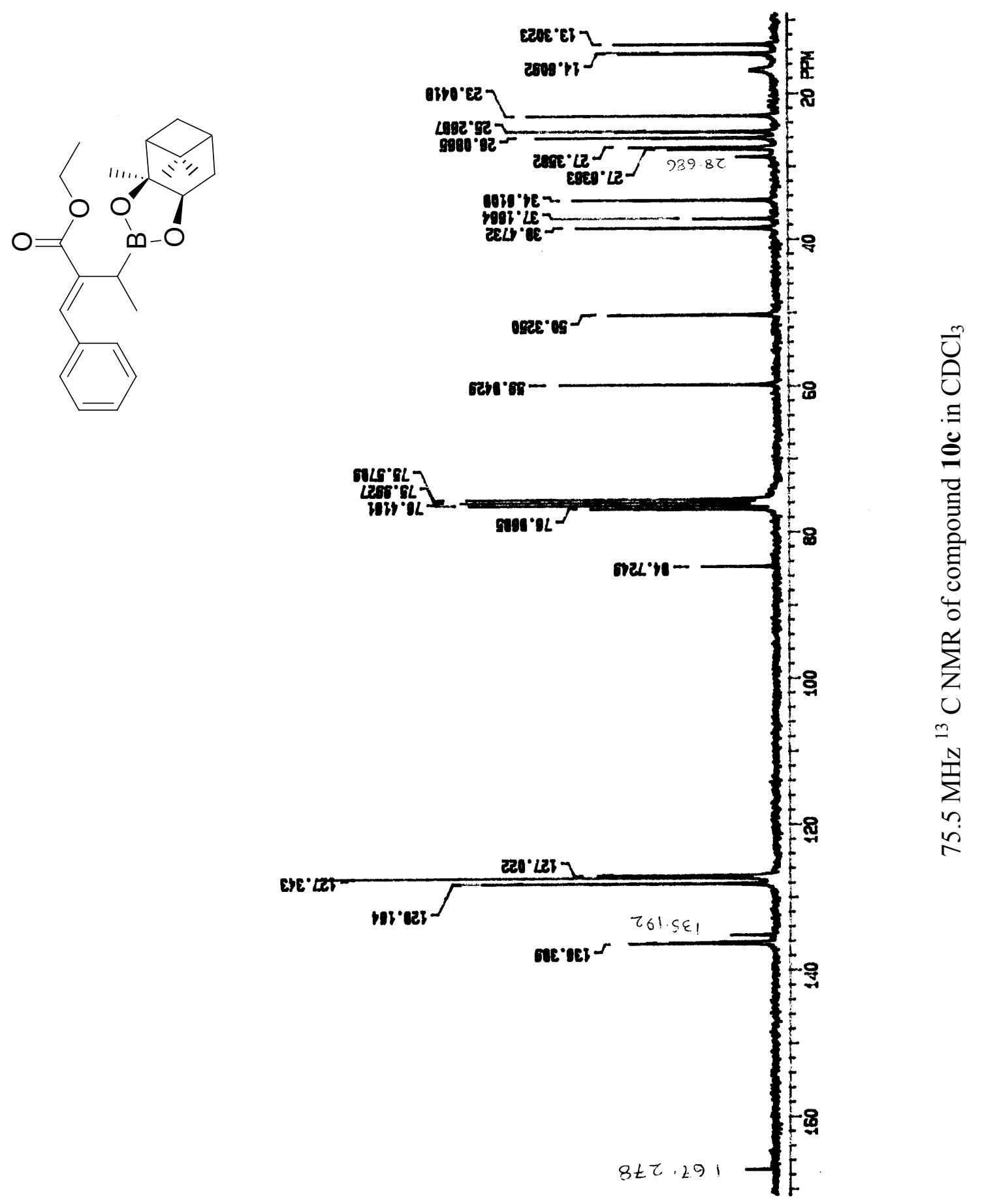

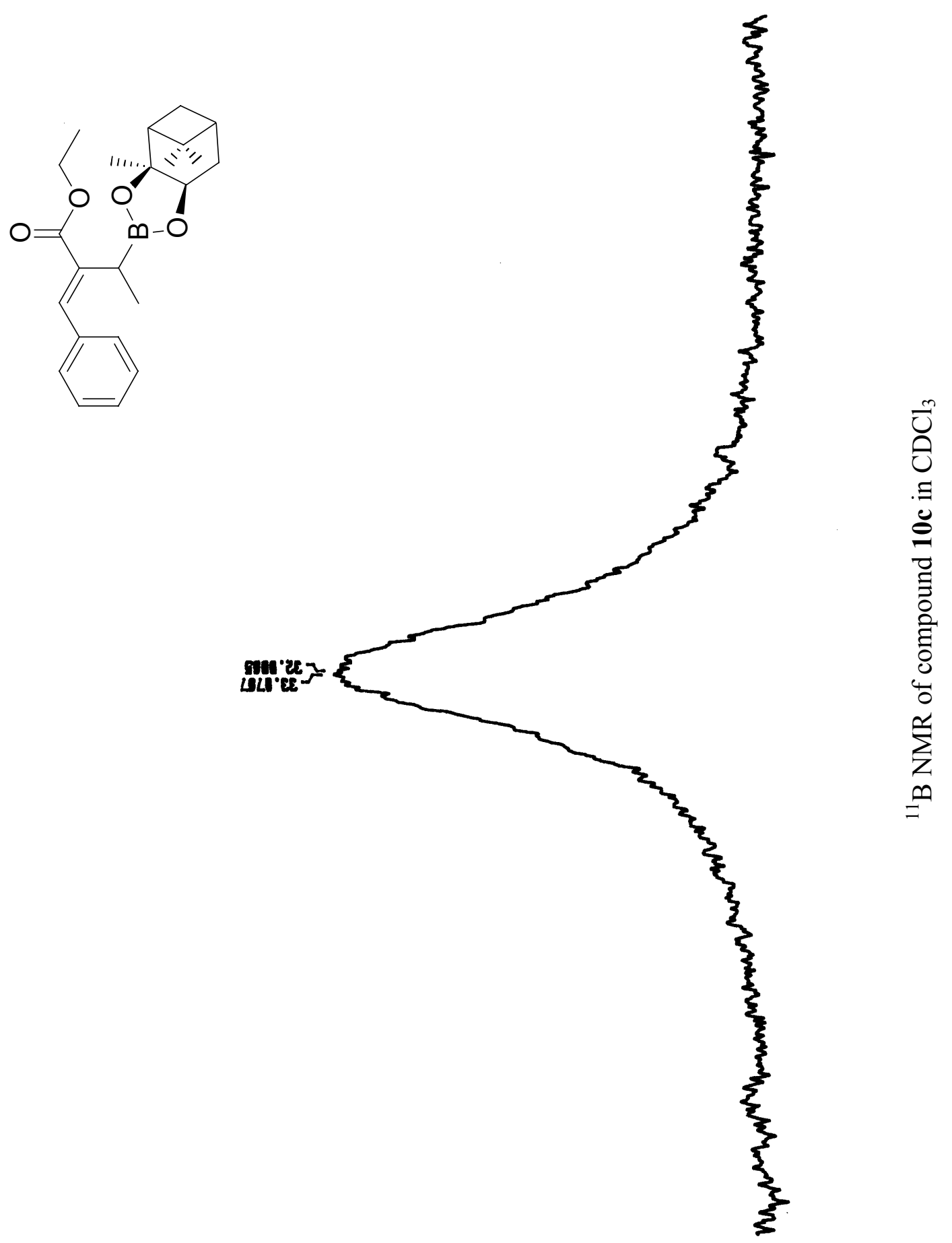


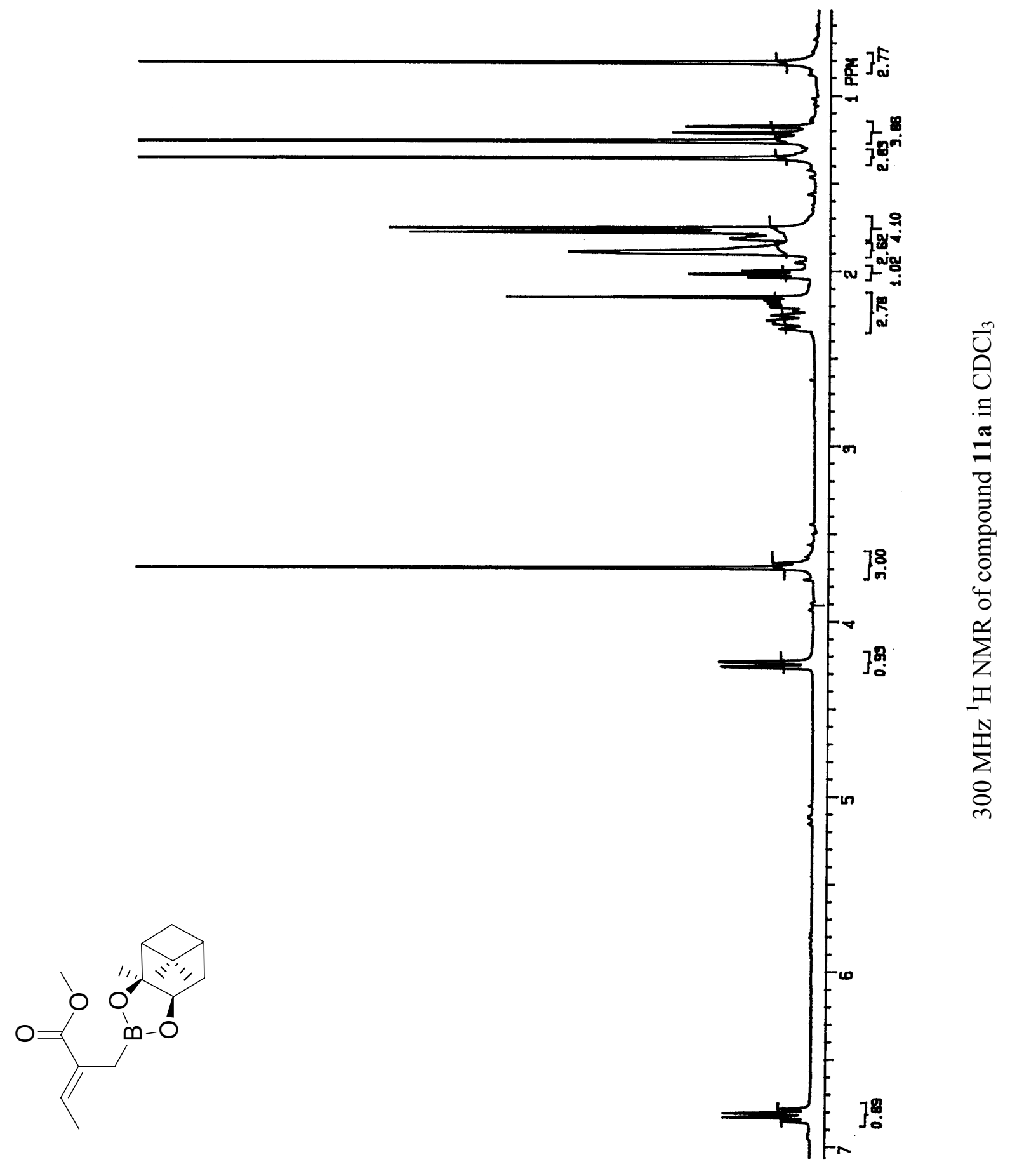




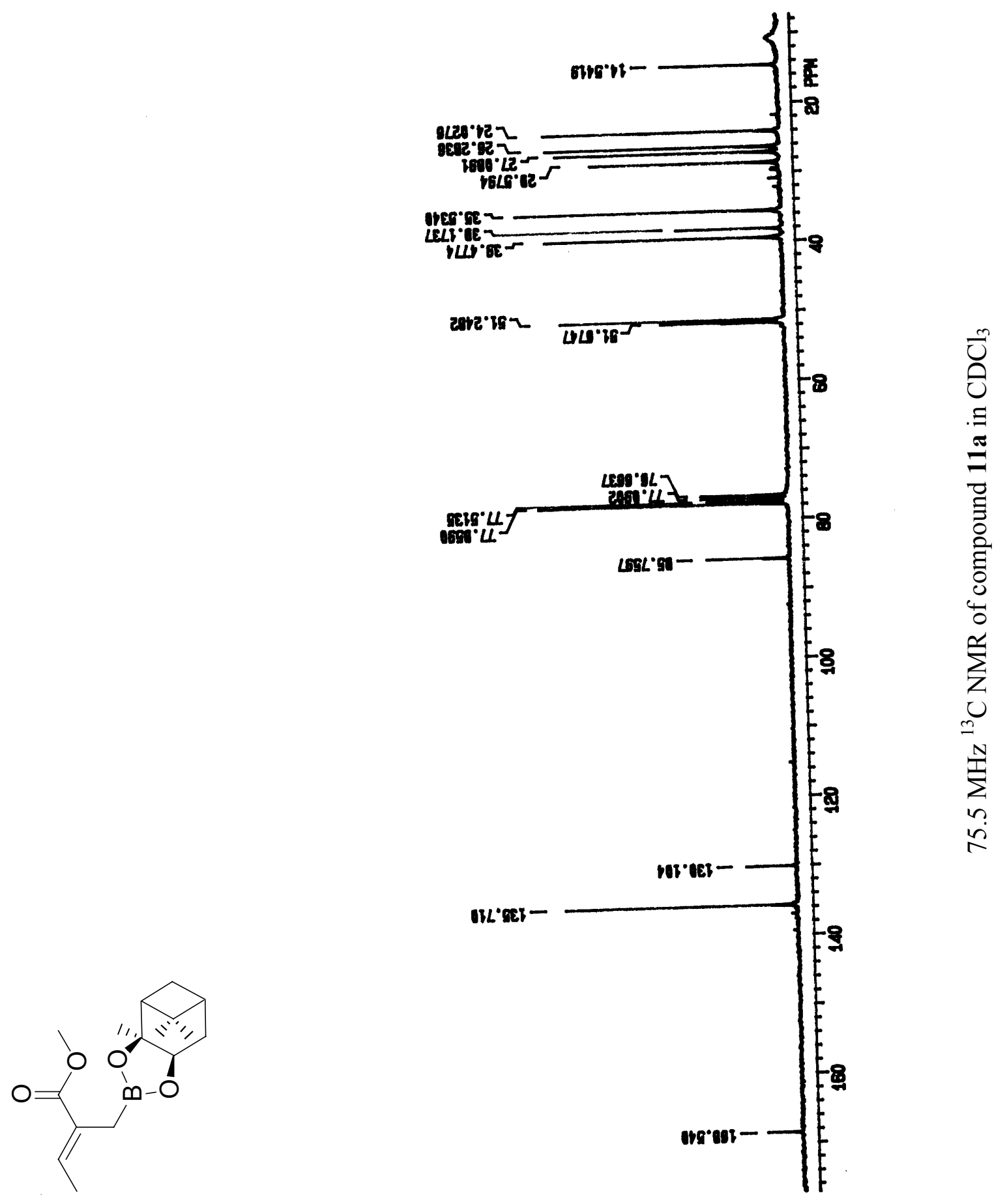




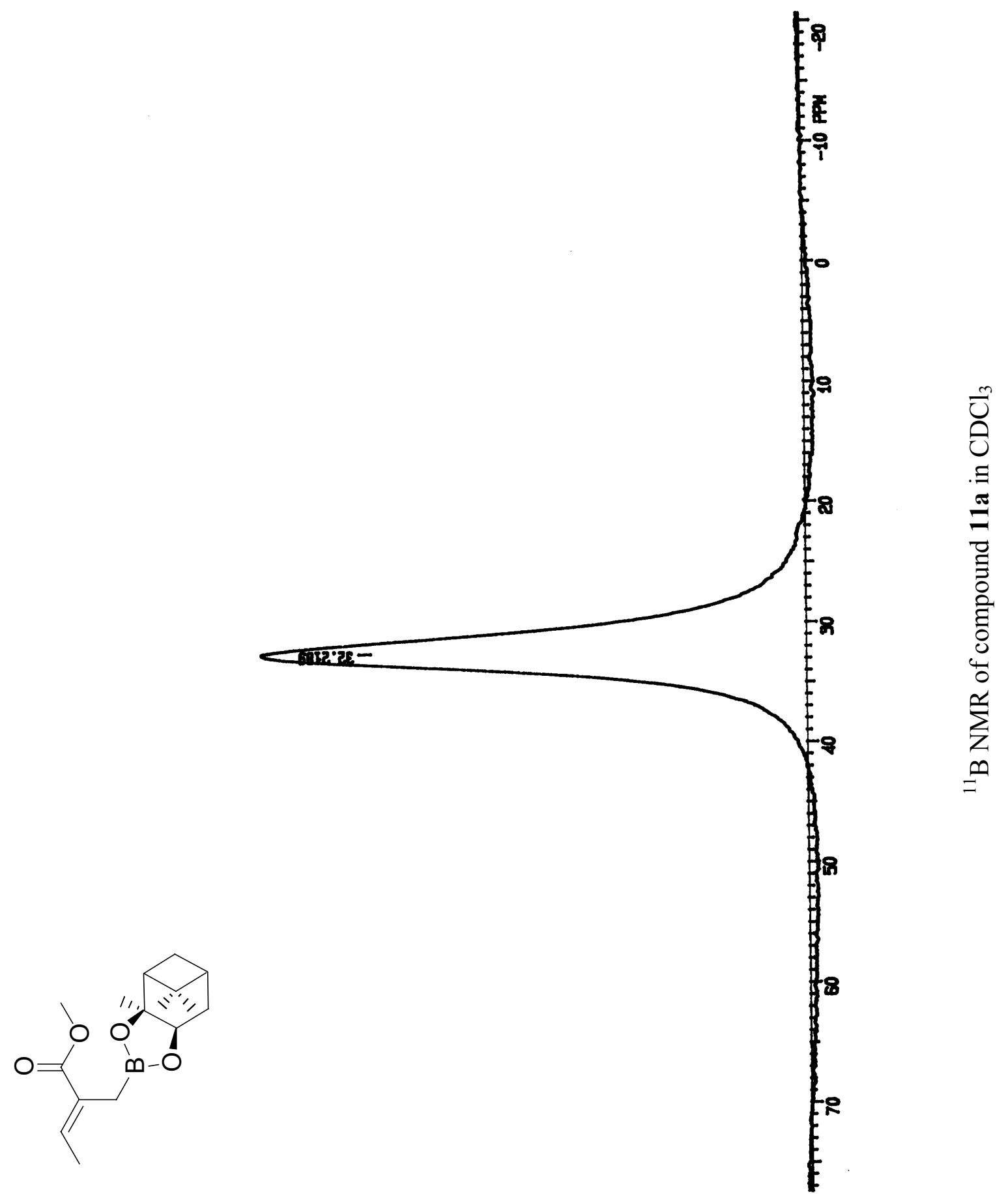




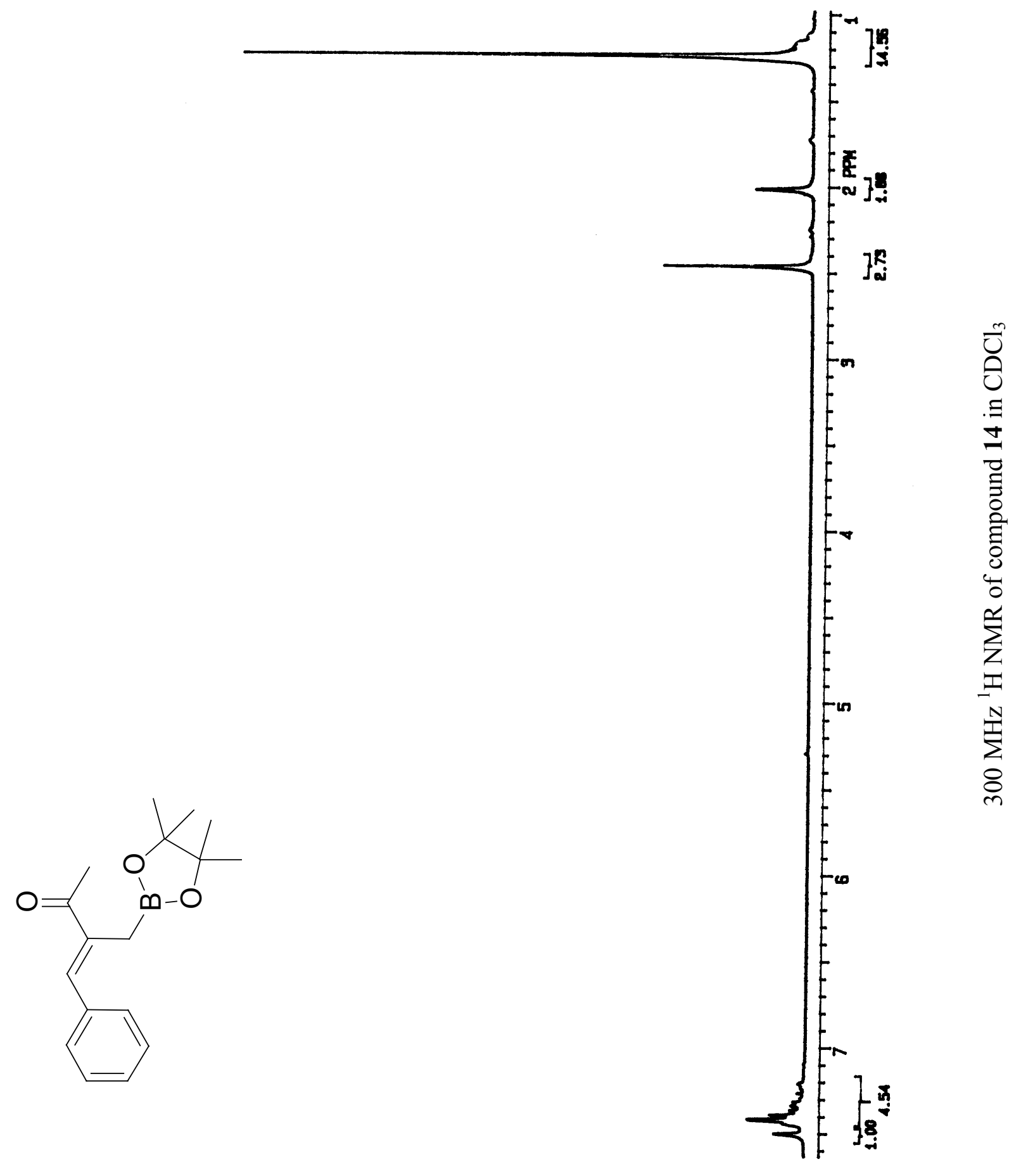




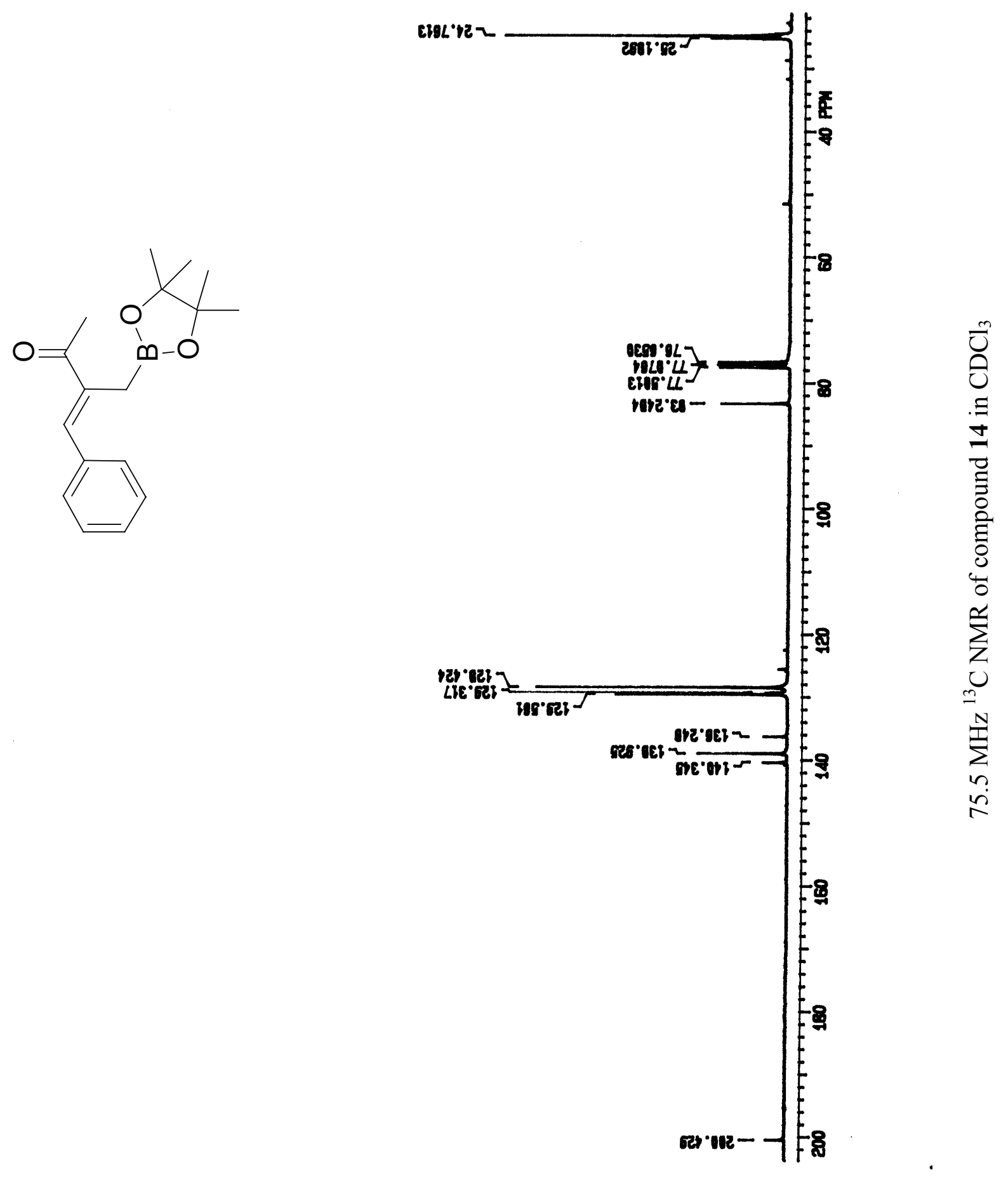




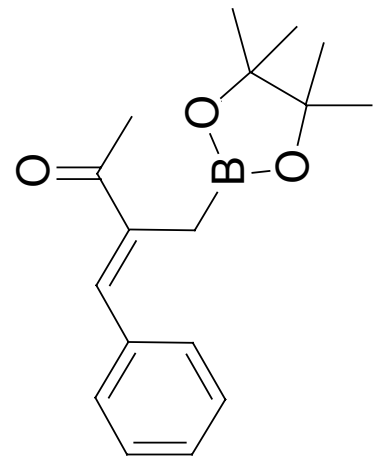

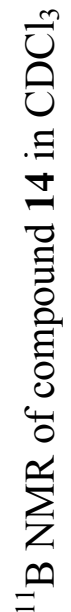




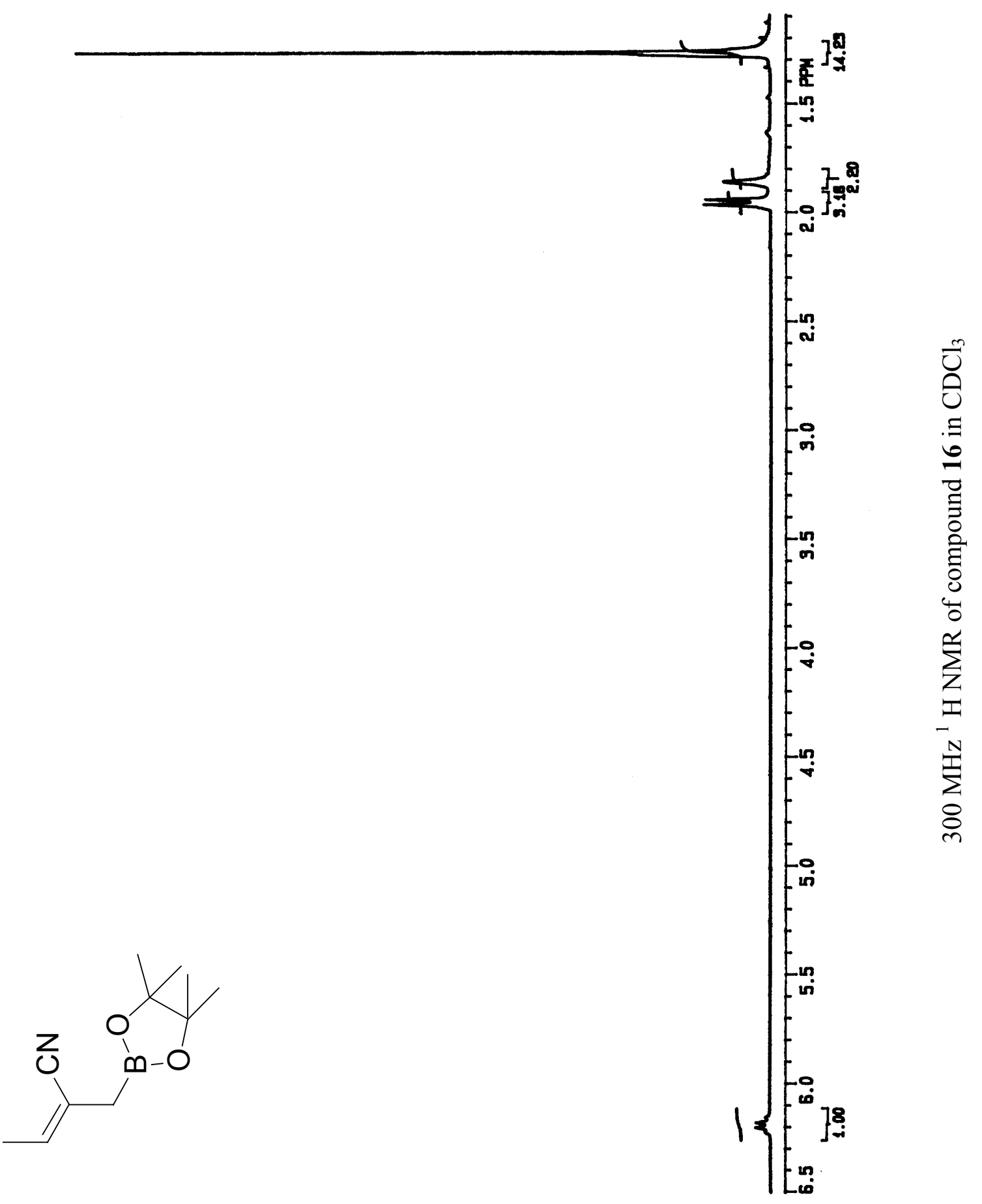




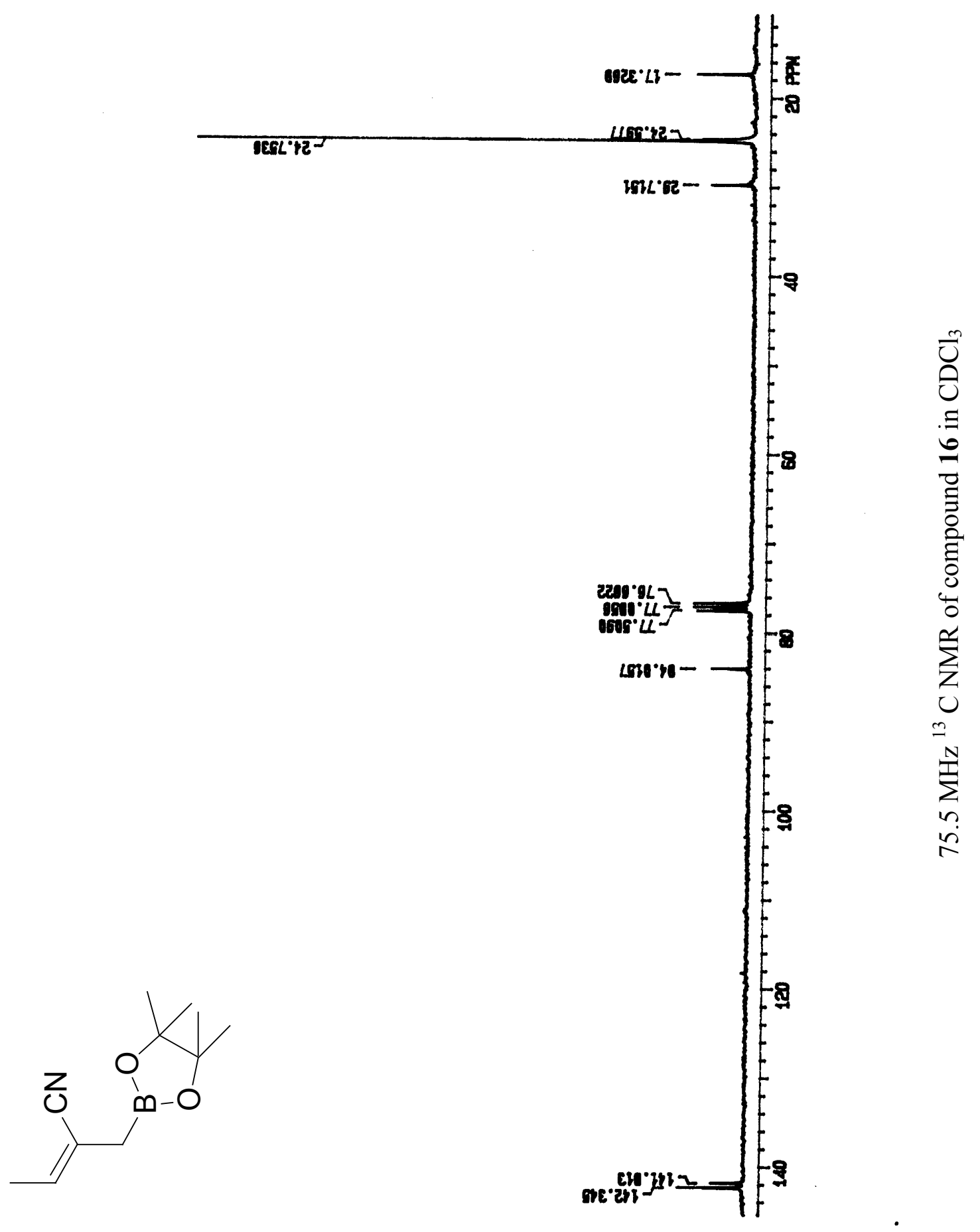




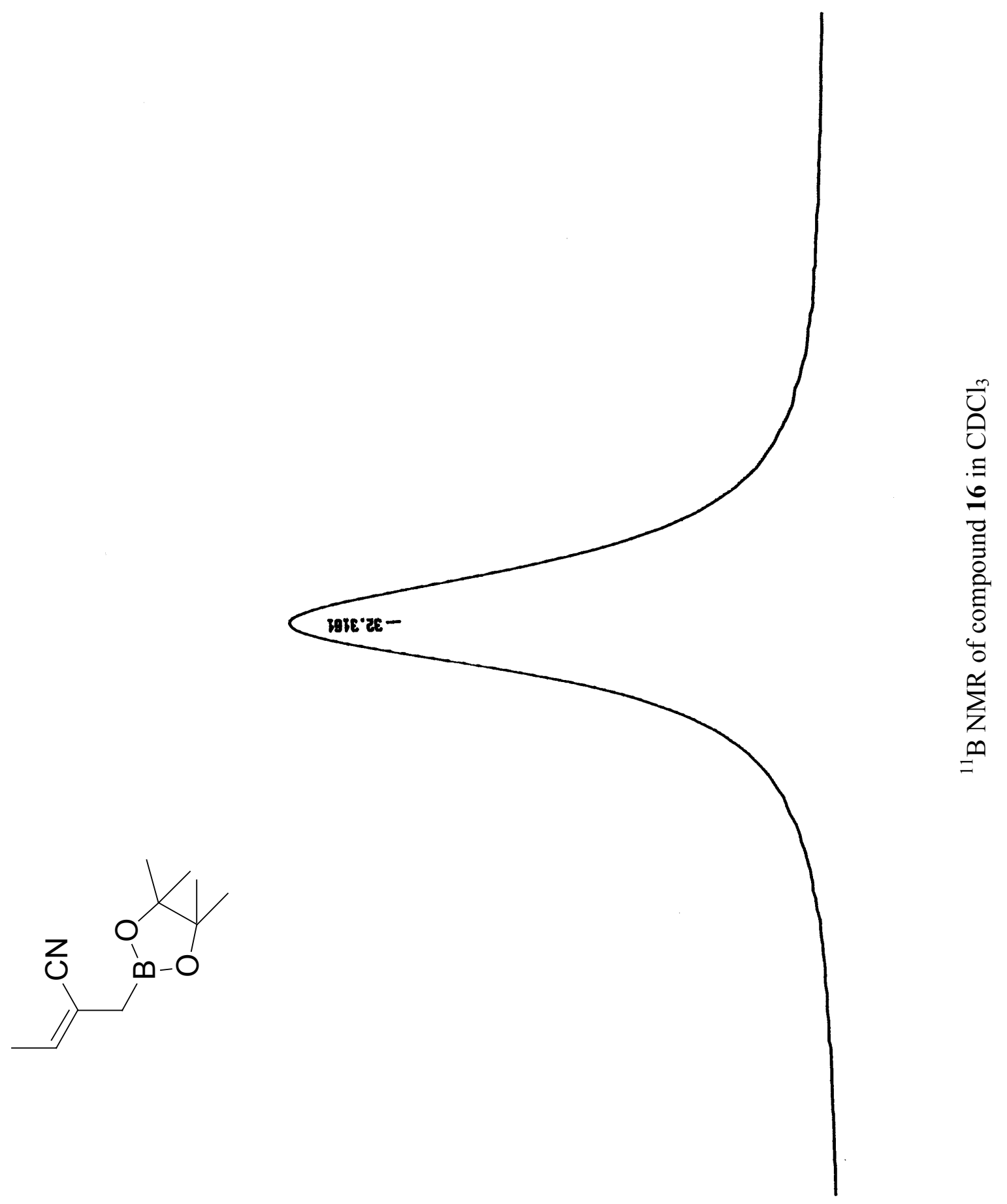




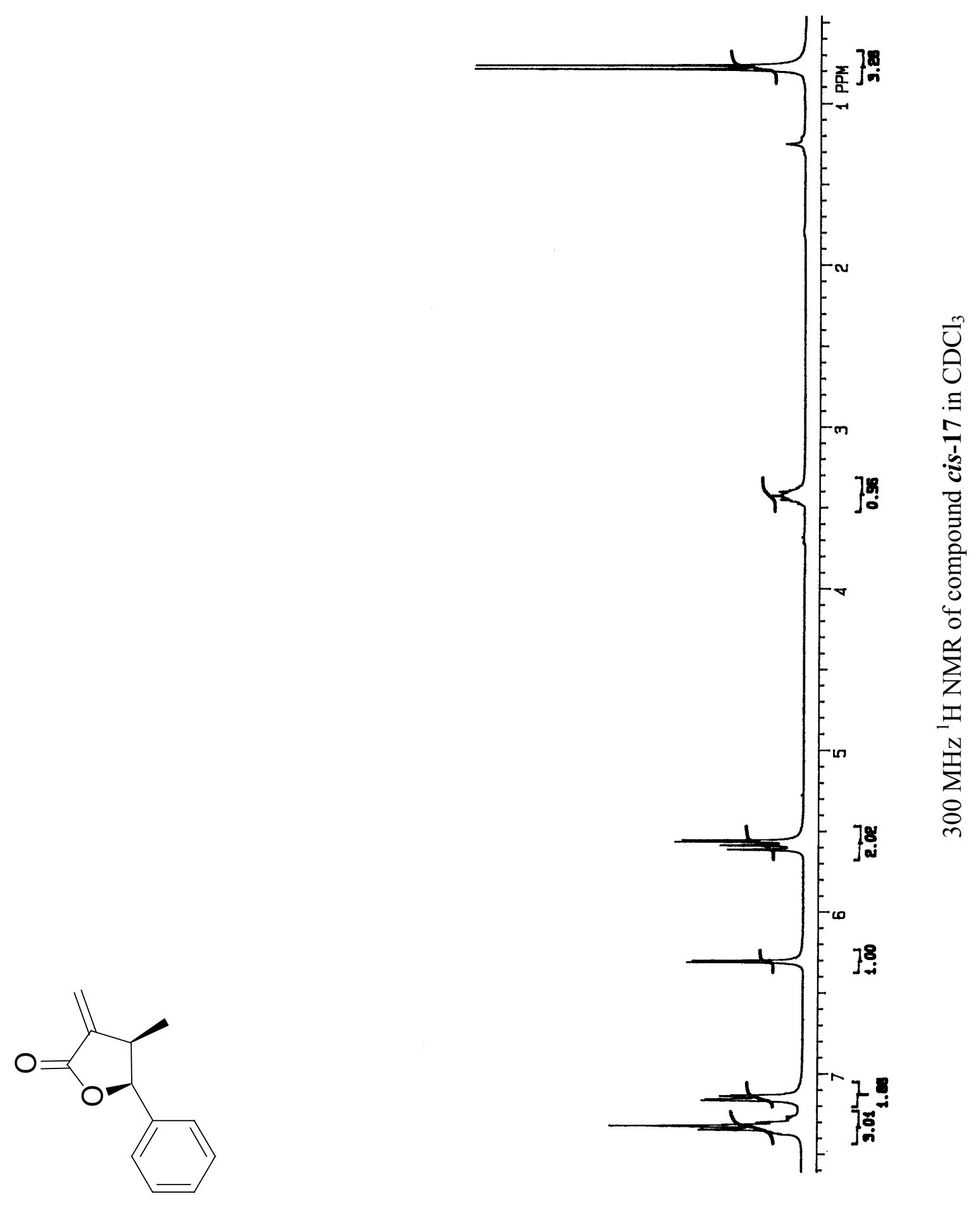



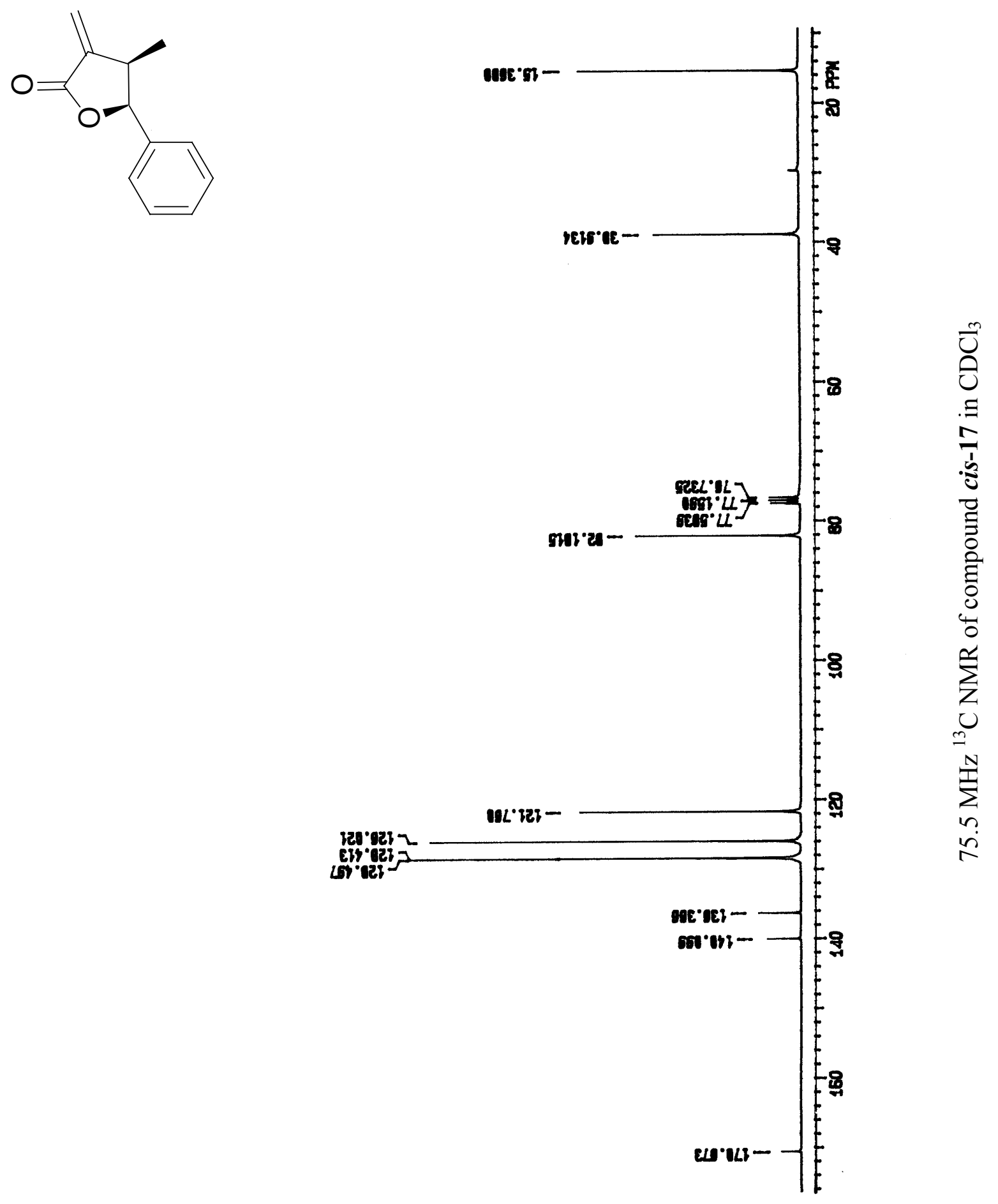